\title{
Contravariant Pseudo-Hessian manifolds and their associated Poisson structures
}

\author{
Abdelhak Abouqateb $^{\mathrm{a}}$, Mohamed Boucetta ${ }^{\mathrm{b}}$, Charif Bourzik ${ }^{\mathrm{c}}$ \\ ${ }^{a}$ Université Cadi-Ayyad \\ Faculté des sciences et techniques \\ BP 549 Marrakech Maroc \\ e-mail: a.abouqateb@uca.ac.ma \\ ${ }^{b}$ Université Cadi-Ayyad \\ Faculté des sciences et techniques \\ BP 549 Marrakech Maroc \\ e-mail: m.boucetta@uca.ac.ma \\ ${ }^{c}$ Université Cadi-Ayyad \\ Faculté des sciences et techniques \\ BP 549 Marrakech Maroc \\ e-mail: bourzikcharif@gmail.com
}

\begin{abstract}
A contravariant pseudo-Hessian manifold is a manifold $M$ endowed with a pair $(\nabla, h)$ where $\nabla$ is a flat connection and $h$ is a symmetric bivector field satisfying a contravariant Codazzi equation. When $h$ is invertible we recover the known notion of pseudo-Hessian manifold. Contravariant pseudo-Hessian manifolds have properties similar to Poisson manifolds and, in fact, to any contravariant pseudo-Hessian manifold $(M, \nabla, h)$ we associate naturally a Poisson tensor on $T M$. We investigate these properties and we study in details many classes of such structures in order to highlight the richness of the geometry of these manifolds.
\end{abstract}

Keywords: Affine manifolds, Poisson manifolds, pseudo-Hessian manifolds, Associative commutative algebras

2000 MSC: 53A15,

2000 MSC: 53D17,

2000 MSC: $17 \mathrm{D} 25$

\section{Introduction}

A contravariant pseudo-Hessian manifold is an affine manifold $(M, \nabla)$ endowed with a symmetric bivector field $h$ such that, for any $\alpha, \beta, \gamma \in \Omega^{1}(M)$,

$$
\left(\nabla_{h_{\sharp}(\alpha)} h\right)(\beta, \gamma)=\left(\nabla_{h_{\sharp}(\beta)} h\right)(\alpha, \gamma),
$$

where $h_{\#}: T^{*} M \longrightarrow T M$ is the contraction. We will refer to (1.1) as contravariant Godazzi equation. These manifolds where introduced in [3] as a generalization of pseudo-Hessian manifolds. Recall that a pseudo-Hessian manifold is an affine manifold $(M, \nabla)$ with a pseudo-Riemannian metric $g$ satisfying the Godazzi equation

$$
\nabla_{X} g(Y, Z)=\nabla_{Y} g(X, Z)
$$


for any $X, Y, Z \in \Gamma(T M)$. The book [13] is devoted to the study of Hessian manifolds which are pseudo-Hessian manifolds with a Riemannian metric.

In this paper, we study contravariant pseudo-Hessian manifolds. The passage from pseudoHessian manifolds to contravariant pseudo-Hessian manifolds is similar to the passage from symplectic manifolds to Poisson manifolds and this similarity will guide our study. Let $(M, \nabla, h)$ be a contravariant pseudo-Hessian manifold. We will show that $T^{*} M$ has a Lie algebroid structure, $M$ has a singular foliation whose leaves are pseudo-Hessian manifolds and $T M$ has a Poisson tensor whose symplectic leaves are pseudo-Kählerian manifolds. We investigate an analog of Darboux-Weinstein's theorem and we show that it is not true in general but holds in some cases. We will study in details the correspondence which maps a contravariant pseudo-Hessian bivector field on $(M, \nabla)$ to a Poisson bivector field on $T M$. We study affine, linear and quadratic contravariant pseudo-Hessian structures on vector spaces and we show that an affine contravariant pseudo-Hessian structure on a vector space $V$ is equivalent to an associative commutative algebra product and a 2-cocycle on $V^{*}$. We study right invariant contravariant pseudo-Hessian structures on a Lie group $G$ and we show that $T G$ has a structure of Lie group (different from the one associated to the adjoint action) for which the associated Poisson tensor is right invariant. We show that a right invariant contravariant pseudo-Hessian structure on a Lie group is equivalent to a $S$-matrix on the associated left symmetric algebra (see [2, 4]) and we associate to any $S$-matrix on a left symmetric algebra $\mathfrak{g}$ a solution of the classical Yang-Baxter equation on $\mathfrak{g} \times \mathfrak{g}$. Finally, we show that an action of a left symmetric algebra $g$ on an affine manifold $(M, \nabla)$ transforms a $S$-matrix on $g$ to a contravariant pseudo-Hessian bivector field on $(M, \nabla)$. Since the Lie algebra of affine vector fields of $(M, \nabla)$ has a natural structure of finite dimensional associative algebra, we have a mean to define contravariant pseudo-Hessian structures on any affine manifold. The paper contains many examples of contravariant pseudo-Hessian structures.

The paper is organized as follows. In Section 2 we give the definition of a contravariant pseudo-Hessian manifold and we investigate its properties. In Section 3 , we study in details the Poisson structure of the tangent bundle of a contravariant pseudo-Hessian manifold. Section 4 is devoted to the study of linear and affine contravariant pseudo-Hessian structures. Quadratic contravariant pseudo-Hessian structures will be studied in Section 5 In Section 6, we study right invariant pseudo-Hessian structures on Lie groups.

\section{Contravariant pseudo-Hessian manifolds: definition and principal properties}

\subsection{Definition of a contravariant pseudo-Hessian manifold}

Recall that an affine manifold is a $n$-manifold $M$ endowed with a maximal atlas such that all transition functions are restrictions of elements of the affine group $\operatorname{Aff}\left(\mathbb{R}^{n}\right)$. This is equivalent to the existence on $M$ of a flat connection $\nabla$, i.e., torsionless and with vanishing curvature (see [13] for more details). An affine coordinates system on an affine manifold $(M, \nabla)$ is a coordinates system $\left(x_{1}, \ldots, x_{n}\right)$ satisfying $\nabla \partial_{x_{i}}=0$ for any $i=1, \ldots, n$.

Let $g$ be a pseudo-Riemannian metric on an affine manifold $(M, \nabla)$. The triple $(M, \nabla, g)$ is called a pseudo-Hessian manifold if $g$ can be locally expressed in any affine coordinates system $\left(x_{1}, \ldots, x_{n}\right)$ as

$$
g_{i j}=\frac{\partial^{2} \phi}{\partial x_{i} \partial x_{j}} .
$$

That is equivalent to $g$ satisfying the Codazzi equation (1.2). When $g$ is Riemannian, we call $(M, \nabla, g)$ a Hessian manifold. The geometry of Hessian manifolds was studied intensively in [13]. 
We consider now a more general situation.

Definition $2.1([3])$. Let $h$ be a symmetric bivector field on an affine manifold $(M, \nabla)$ and $h_{\sharp}$ : $T^{*} M \rightarrow T M$ the associated contraction given by $\beta\left(h_{\sharp}(\alpha)\right)=h(\alpha, \beta)$. The triple $(M, \nabla, h)$ is called a contravariant pseudo-Hessian manifold if $h$ satisfies the contravariant Codazzi equation

$$
\left(\nabla_{h_{\sharp}(\alpha)} h\right)(\beta, \gamma)=\left(\nabla_{h_{\sharp}(\beta)} h\right)(\alpha, \gamma),
$$

for any $\alpha, \beta, \gamma \in \Omega^{1}(M)$. We call such h a pseudo-Hessian bivector field.

One can see easily that if $(M, \nabla, g)$ is a pseudo-Hessian manifold then $\left(M, \nabla, g^{-1}\right)$ is a contravariant pseudo-Hessian manifold.

The following proposition is obvious and gives the local expression of the equation (2.1) in affine charts.

Proposition 2.2. Let $(M, \nabla, h)$ be an affine manifold endowed with a symmetric bivector field. Then $h$ satisfies (2.1) if and only if, for any $m \in M$, there exists an affine coordinates system $\left(x_{1}, \ldots, x_{n}\right)$ around $m$ such that for any $1 \leq i<j \leq n$ and any $k=1, \ldots, n$

$$
\sum_{l=1}^{n}\left[h_{i l} \partial_{x_{l}}\left(h_{j k}\right)-h_{j l} \partial_{x_{l}}\left(h_{i k}\right)\right]=0,
$$

where $h_{i j}=h\left(d x_{i}, d x_{j}\right)$.

Example 2.3. 1. Take $M=\mathbb{R}^{n}$ endowed with its canonical affine structure and consider

$$
h=\sum_{i=1}^{n} f_{i}\left(x_{i}\right) \partial_{x_{i}} \otimes \partial_{x_{i}},
$$

where $f_{i}: \mathbb{R} \longrightarrow \mathbb{R}$ for $i=1, \ldots, n$. Then one can see easily that $h$ satisfies (2.2) and hence defines a contravariant pseudo-Hessian structure on $\mathbb{R}^{n}$.

2. Take $M=\mathbb{R}^{n}$ endowed with its canonical affine structure and consider

$$
h=\sum_{i, j=1}^{n} x_{i} x_{j} \partial_{x_{i}} \otimes \partial_{x_{j}} .
$$

Then one can see easily that $h$ satisfies (2.2) and hence defines a contravariant pseudoHessian structure on $\mathbb{R}^{n}$.

3. Let $(M, \nabla)$ be an affine manifold, $\left(X_{1}, \ldots, X_{r}\right)$ a family of parallel vector fields and $\left(a_{i, j}\right)_{1 \leq i, j \leq n}$ a symmetric n-matrix. Then

$$
h=\sum_{i, j} a_{i, j} X_{i} \otimes X_{j}
$$

defines a contravariant pseudo-Hessian structure on $M$.

\subsection{The Lie algebroid of a contravariant pseudo-Hessian manifold}

We show that associated to any contravariant pseudo-Hessian manifold there is a Lie algebroid structure on its cotangent bundle and a Lie algebroid flat connection. The reader can consult [10, 12] for more details on Lie algebroids and their connections. 
Let $(M, \nabla, h)$ be an affine manifold endowed with a symmetric bivector field. We associate to this triple a bracket on $\Omega^{1}(M)$ by putting

$$
[\alpha, \beta]_{h}:=\nabla_{h_{\sharp}(\alpha)} \beta-\nabla_{h_{\sharp}(\beta)} \alpha,
$$

and a map $\mathcal{D}: \Omega^{1}(M) \times \Omega^{1}(M) \longrightarrow \Omega^{1}(M)$ given by

$$
<\mathcal{D}_{\alpha} \beta, X>:=\left(\nabla_{X} h\right)(\alpha, \beta)+<\nabla_{h_{\sharp}(\alpha)} \beta, X>,
$$

for any $\alpha, \beta \in \Omega^{1}(M)$ and $X \in \Gamma(T M)$. This bracket is skew-symmetric and satisfies obviously

$$
[\alpha, \beta]_{h}=\mathcal{D}_{\alpha} \beta-\mathcal{D}_{\beta} \alpha \quad \text { and } \quad[\alpha, f \beta]_{h}=f[\alpha, \beta]_{h}+h_{\#}(\alpha)(f) \beta,
$$

where $f \in C^{\infty}(M), \alpha, \beta \in \Omega^{1}(M)$.

Theorem 2.4. With the hypothesis and notations above, the following assertions are equivalent:

(i) $h$ is a pseudo-Hessian bivector field.

(ii) $\left(T^{*} M, h_{\sharp},[,]_{h}\right)$ is a Lie algebroid.

In this case, $\mathcal{D}$ is a connection for the Lie algebroid structure $\left.\left(T^{*} M, h_{\#},[,]\right]_{h}\right)$ satisfying

$$
h_{\#}\left(\mathcal{D}_{\alpha} \beta\right)=\nabla_{h_{\sharp}(\alpha)} h_{\#}(\beta) \quad \text { and } \quad R_{\mathcal{D}}(\alpha, \beta):=\mathcal{D}_{[\alpha, \beta]_{h}}-\mathcal{D}_{\alpha} \mathcal{D}_{\beta}+\mathcal{D}_{\beta} \mathcal{D}_{\alpha}=0
$$

for any $\alpha, \beta \in \Omega^{1}(M)$.

Proof. According to [3, Proposition 2.1], $\left(T^{*} M, h_{\#},[,]_{h}\right)$ is a Lie algebroid if and only if, for any affine coordinates system $\left(x_{1}, \ldots, x_{n}\right)$,

$$
h_{\#}\left(\left[d x_{i}, d x_{j}\right]_{h}\right)=\left[h_{\#}\left(d x_{i}\right), h_{\#}\left(d x_{j}\right)\right] \quad \text { and } \quad \oint_{i, j, k}\left[d x_{i},\left[d x_{j}, d x_{k}\right]_{h}\right]_{h}=0,
$$

for $1 \leq i<j<k \leq n$. Since $\left[d x_{i}, d x_{j}\right]_{h}=0$ this is equivalent to $\left[h_{\#}\left(d x_{i}\right), h_{\#}\left(d x_{j}\right)\right]=0$ for any $1 \leq i<j \leq n$ which is equivalent to (2.2).

Suppose now that (i) or (ii) holds. For any, $\alpha, \beta, \gamma \in \Omega^{1}(M)$,

$$
\begin{aligned}
<\mathcal{D}_{\alpha} \beta, h_{\#}(\gamma)> & =\nabla_{h_{\sharp}(\gamma)} h(\alpha, \beta)+h\left(\nabla_{h_{*}(\alpha)}^{*} \beta, \gamma\right) \\
& =\nabla_{h_{\sharp}(\alpha)} h(\gamma, \beta)+h\left(\nabla_{h_{\sharp}(\alpha)}^{*} \beta, \gamma\right) \\
& =h_{\#}(\alpha) \cdot h(\beta, \gamma)-h\left(\nabla_{h_{\sharp}(\alpha)}^{*} \gamma, \beta\right) \\
& =<\gamma, \nabla_{h_{\sharp}(\alpha)} h_{\#}(\beta)>.
\end{aligned}
$$

This shows that $h_{\#}\left(\mathcal{D}_{\alpha} \beta\right)=\nabla_{h_{\sharp}(\alpha)} h_{\#}(\beta)$.

Let us show now that the curvature of $\mathcal{D}$ vanishes. Since $\left[d x_{i}, d x_{j}\right]_{h}=0$, it suffices to show that, for any $i, j, k \in\{1, \ldots, n\}$ with $i<j, \mathcal{D}_{d x_{i}} \mathcal{D}_{d x_{j}} d x_{k}=\mathcal{D}_{d x_{j}} \mathcal{D}_{d x_{i}} d x_{k}$. We have

$$
\prec \mathcal{D}_{d x_{i}} d x_{k}, \frac{\partial}{\partial x_{l}}>=\frac{\partial h_{i k}}{\partial x_{l}}
$$

and hence

$$
\mathcal{D}_{d x_{i}} d x_{k}=\sum_{l=1}^{n} \frac{\partial h_{i k}}{\partial x_{l}} d x_{l}
$$


and then

$$
\begin{aligned}
\mathcal{D}_{d x_{j}} \mathcal{D}_{d x_{i}} d x_{k} & =\sum_{l=1}^{n} \mathcal{D}_{d x_{j}}\left(\frac{\partial h_{i k}}{\partial x_{l}} d x_{l}\right) \\
& =\sum_{l=1}^{n}\left(h_{\sharp}\left(d x_{j}\right)\left(\frac{\partial h_{i k}}{\partial x_{l}}\right) d x_{l}+\frac{\partial h_{i k}}{\partial x_{l}}\left(\sum_{s=1}^{n} \frac{\partial h_{j l}}{\partial x_{s}} d x_{s}\right)\right) \\
& =\sum_{l, r} h_{j r}\left(\frac{\partial^{2} h_{i k}}{\partial x_{r} \partial x_{l}}\right) d x_{l}+\sum_{s, l} \frac{\partial h_{i k}}{\partial x_{l}} \frac{\partial h_{j l}}{\partial x_{s}} d x_{s} \\
& =\sum_{l, r} h_{j r}\left(\frac{\partial^{2} h_{i k}}{\partial x_{r} \partial x_{l}}\right) d x_{l}+\sum_{l, r} \frac{\partial h_{i k}}{\partial x_{r}} \frac{\partial h_{j r}}{\partial x_{l}} d x_{l} \\
& =\sum_{l, r}\left(h_{j r}\left(\frac{\partial^{2} h_{i k}}{\partial x_{r} \partial x_{l}}\right)+\frac{\partial h_{i k}}{\partial x_{r}} \frac{\partial h_{j r}}{\partial x_{l}}\right) d x_{l} \\
& =\sum_{l} \frac{\partial}{\partial x_{l}}\left(\sum_{r} h_{j r} \frac{\partial h_{i k}}{\partial x_{r}}\right) d x_{l} .
\end{aligned}
$$

So

$$
\left.\mathcal{D}_{d x_{i}} \mathcal{D}_{d x_{j}} d x_{k}-\mathcal{D}_{d x_{j}} \mathcal{D}_{d x_{i}} d x_{k}=d\left(\sum_{r}\left(h_{j r} \frac{\partial h_{i k}}{\partial x_{r}}-h_{i r} \frac{\partial h_{j k}}{\partial x_{r}}\right)\right)\right) \stackrel{[2.2]}{=} d(0)=0 .
$$

The following result is an important consequence of Theorem 2.4

Proposition 2.5. ([4, Theorem 6.7]) Let $(M, \nabla, h)$ be a contravariant pseudo-Hessian manifold. Then:

1. The distribution $\operatorname{Im} h_{\#}$ is integrable and defines a singular foliation $\mathcal{L}$ on $M$.

2. For any leaf $L$ of $\mathcal{L},\left(L, \nabla_{\mid L}, g_{L}\right)$ is a pseudo-Hessian manifold where $g_{L}$ is given by $g_{L}\left(h_{\#}(\alpha), h_{\#}(\beta)\right)=$ $h(\alpha, \beta)$.

We will call the foliation defined by $\operatorname{Im} h_{\sharp}$ the affine foliation associated to $(M, \nabla, h)$.

Remark 2.6. This proposition shows that contravariant pseudo-Hessian bivector fields can be used either to build examples of affine foliations on affine manifolds or to build examples of pseudo-Hessian manifolds.

For the reader familiar with Poisson manifolds what we have established so far shows the similarities between Poisson manifolds and contravariant pseudo-Hessian manifolds. One can consult [8] for more details on Poisson geometry. Poisson manifolds have many relations with Lie algebras and we will see now and in Section 4 that contravariant pseudo-Hessian manifolds are related to commutative associative algebras.

Let $(M, \nabla, h)$ be a contravariant pseudo-Hessian manifold and $\mathcal{D}$ the connection given in (2.4). Let $x \in M$ and $\mathfrak{g}_{x}=\operatorname{ker} h_{\#}(x)$. For any $\alpha, \beta \in \Omega^{1}(M), h_{\#}\left(\mathcal{D}_{\alpha} \beta\right)=\nabla_{h_{\sharp}(\alpha)} h_{\#}(\beta)$. This shows that if $h_{\#}(\alpha)(x)=0$ then $h_{\#}\left(\mathcal{D}_{\alpha} \beta\right)(x)=0$. Moreover, $\mathcal{D}_{\alpha} \beta-\mathcal{D}_{\beta} \alpha=\nabla_{h_{\sharp}(\alpha)} \beta-\nabla_{h_{\sharp}(\beta)} \alpha$. This implies that if $h_{\#}(\alpha)(x)=h_{\#}(\beta)(x)=0$ then $\mathcal{D}_{\alpha} \beta(x)=\mathcal{D}_{\beta} \alpha(x)$. For any $a, b \in \mathfrak{g}_{x}$ put

$$
a \bullet b=\left(\mathcal{D}_{\alpha} \beta\right)(x),
$$


where $\alpha, \beta$ are two differential 1-forms satisfying $\alpha(x)=a$ and $\beta(x)=b$. This defines a commutative product on $\mathfrak{g}_{x}$ and moreover, by using the vanishing of the curvature of $\mathcal{D}$, we get:

Proposition 2.7. $\left(\mathfrak{g}_{x}, \bullet\right)$ is a commutative associative algebra.

Near a point where $h$ vanishes, the algebra structure of $\mathfrak{g}_{x}$ can be made explicit.

Proposition 2.8. We consider $\mathbb{R}^{n}$ endowed with its canonical affine connection, $h$ a symmetric bivector field on $\mathbb{R}^{n}$ such that $h(0)=0$ and $\left(\mathbb{R}^{n}, \nabla, h\right)$ is a contravariant pseudo-Hessian manifold. Then the product on $\left(\mathbb{R}^{n}\right)^{*}$ given by

$$
e_{i}^{*} \bullet e_{j}^{*}=\sum_{k=1}^{n} \frac{\partial h_{i j}}{\partial x_{k}}(0) e_{k}^{*}
$$

is associative and commutative.

Proof. It is a consequence of the relation $\mathcal{D}_{d x_{i}} d x_{j}=d h_{i j}$ true by virtue of (2.4).

\subsection{The product of contravariant pseudo-Hessian manifolds and the splitting theorem}

As the product of two Poisson manifolds is a Poisson manifold [15], the product of two contravariant pseudo-Hessian manifolds is a contravariant pseudo-Hessian manifold.

Let $\left(M_{1}, \nabla^{1}, h^{1}\right)$ and $\left(M_{2}, \nabla^{2}, h^{2}\right)$ be two contravariant pseudo-Hessian manifolds. We denote by $p_{i}: M=M_{1} \times M_{2} \rightarrow M_{i}, i=1,2$ the canonical projections. For any $X \in \Gamma\left(T M_{1}\right)$ and $Y \in \Gamma\left(T M_{2}\right)$, we denote by $X+Y$ the vector field on $M$ given by $(X+Y)\left(m_{1}, m_{2}\right)=\left(X\left(m_{1}\right), Y\left(m_{2}\right)\right)$. The product of the affine atlases on $M_{1}$ and $M_{2}$ is an affine atlas on $M$ and the corresponding affine connection is the unique flat connection $\nabla$ on $M$ satisfying $\nabla_{X_{1}+Y_{1}}\left(X_{2}+Y_{2}\right)=\nabla_{X_{1}}^{1} Y_{1}+\nabla_{X_{2}}^{2} Y_{2}$, for any $X_{1}, X_{2} \in \Gamma\left(T M_{1}\right)$ and $Y_{1}, Y_{2} \in \Gamma\left(T M_{2}\right)$. Moreover, the product of $h_{1}$ and $h_{2}$ is the unique symmetric bivector field $h$ satisfying

$$
h\left(p_{1}^{*} \alpha_{1}, p_{1}^{*} \alpha_{2}\right)=h^{1}\left(\alpha_{1}, \alpha_{2}\right) \circ p_{1}, h\left(p_{2}^{*} \beta_{1}, p_{2}^{*} \beta_{2}\right)=h^{2}\left(\beta_{1}, \beta_{2}\right) \circ p_{2} \quad \text { and } \quad h\left(p_{1}^{*} \alpha_{1}, p_{2}^{*} \beta_{1}\right)=0 \text {, }
$$

for any $\alpha_{1}, \beta_{1} \in \Omega^{1}\left(M_{1}\right), \alpha_{2}, \beta_{2} \in \Omega^{1}\left(M_{2}\right)$,

Proposition 2.9. $(M, \nabla, h)$ is a contravariant pseudo-Hessian manifold.

Proof. Let $\left(m_{1}, m_{2}\right) \in M$. Choose an affine coordinates system $\left(x_{1}, \ldots, x_{n_{1}}\right)$ near $m_{1}$ and an affine coordinates system $\left(y_{1}, \ldots, y_{n_{2}}\right)$ near $m_{2}$. Then

$$
h=\sum_{i, j} h_{i j}^{1} \circ p_{1} \partial_{x_{i}} \otimes \partial_{x_{j}}+\sum_{l, k} h_{l k}^{2} \circ p_{2} \partial_{y_{l}} \otimes \partial_{y_{k}}
$$

and one can check easily that $h$ satisfies (2.2).

If we pursue the exploration of the analogies between Poisson manifolds and contravariant pseudo-Hessian manifolds we can ask naturally if there is an analog of the Darboux-Weinstein's theorem (see[15]) in the context of contravariant pseudo-Hessian manifolds. More precisely, let $(M, \nabla, h)$ be a contravariant pseudo-Hessian manifold and $m \in M$ where $\operatorname{rank} h_{\#}(m)=r$. One can ask if there exits an affine coordinates system $\left(x_{1}, \ldots, x_{r}, y_{1}, \ldots, y_{n-r}\right)$ such that

$$
h=\sum_{i, j=1}^{r} h_{i j}\left(x_{1}, \ldots, x_{r}\right) \partial_{x_{i}} \otimes \partial_{x_{j}}+\sum_{i, j=1}^{n-r} f_{i j}\left(y_{1}, \ldots, y_{n-r}\right) \partial_{y_{i}} \otimes \partial_{y_{j}},
$$


where $\left(h_{i j}\right)_{1 \leq i, j \leq r}$ is invertible and its the inverse of $\left(\frac{\partial^{2} \phi}{\partial x_{i} \partial x_{j}}\right)_{1 \leq i, j \leq r}$ and $f_{i j}(m)=0$ for any $i, j$. Moreover, if the rank of $h_{\#}$ is constant near $m$ then the functions $f_{i j}$ vanish.

The answer is no in general for a geometric reason. Suppose that $m$ is regular, i.e., the rank of $h$ is constant near $m$ and suppose that there exists an affine coordinates system $\left(x_{1}, \ldots, x_{r}, y_{1}, \ldots, y_{n-r}\right)$ such that

$$
h=\sum_{i, j=1}^{r} h_{i j}\left(x_{1}, \ldots, x_{r}\right) \partial_{x_{i}} \otimes \partial_{x_{j}} .
$$

This will have a strong geometric consequence, namely that $\operatorname{Im} h_{\#}=\operatorname{span}\left(\partial_{x_{1}}, \ldots, \partial_{x_{r}}\right)$ and the associated affine foliation is parallel, i.e., if $X$ is a local vector field and $Y$ is tangent to the foliation then $\nabla_{X} Y$ is tangent to the foliation. We give now an example of a regular contravariant pseudo-Hessian manifold whose associated affine foliation is not parallel which shows that the analog of Darboux-Weinstein is not true in general.

Example 2.10. We consider $M=\mathbb{R}^{4}$ endowed with its canonical affine connection $\nabla$, denote by $(x, y, z, t)$ its canonical coordinates and consider

$$
X=\cos (t) \partial_{x}+\sin (t) \partial_{y}+\partial_{z}, Y=-\sin (t) \partial_{x}+\cos (t) \partial_{y} \quad \text { and } \quad h=X \otimes Y+Y \otimes X .
$$

We have $\nabla_{X} X=\nabla_{Y} X=\nabla_{X} Y=\nabla_{Y} Y=0$ and hence $h$ is a pseudo-Hessian bivector field, $\operatorname{Im} h_{\#}=\operatorname{span}\{X, Y\}$ and the rank of $h$ is constant equal to 2 . However, the foliation associated to $\operatorname{Im} h_{\#}$ is not parallel since $\nabla_{\partial_{t}} Y=-X+\partial_{z} \notin \operatorname{Im} h_{\#}$.

However, when $h$ has constant rank equal to $\operatorname{dim} M-1$, we have the following result and its important corollary.

Theorem 2.11. Let $(M, \nabla, h)$ be a contravariant pseudo-Hessian manifold and $m \in M$ such that $m$ is a regular point and the rank of $h_{\#}(m)$ is equal to $n-1$. Then there exists an affine coordinates system $\left(x_{1}, \ldots, x_{n}\right)$ around $m$ and a function $f\left(x_{1}, \ldots, x_{n}\right)$ such that

$$
h=\sum_{i, j=1}^{n-1} h_{i j} \partial_{x_{i}} \otimes \partial_{x_{j}},
$$

and the matrix $\left(h_{i j}\right)_{1 \leq i, j \leq n-1}$ is invertible and its inverse is the matrix $\left(\frac{\partial^{2} f}{\partial x_{i} \partial x_{j}}\right)_{1 \leq i, j \leq n-1}$.

Corollary 2.12. Let $(M, \nabla, h)$ be a contravariant pseudo-Hessian manifold with $h$ of constant rank equal to $\operatorname{dim} M-1$. Then the affine foliation associated to $\operatorname{Im} h_{\#}$ is $\nabla$-parallel.

In order to prove this theorem, we need the following lemma.

Lemma 2.13. Let $f: \mathbb{R}^{2} \longrightarrow \mathbb{R}$ be a differentiable function such that $\partial_{x}(f)+f \partial_{y}(f)=0$. Then $f$ is a constant.

Proof. Let $f$ be a solution of the equation above. We consider the vector field $X_{f}=\partial_{x}+f \partial_{y}$. The integral curve $(x(t), y(t))$ of $X_{f}$ passing through $(a, b) \in \mathbb{R}^{2}$ satisfies

$$
x^{\prime}(t)=1, \quad y^{\prime}(t)=f(x(t), y(t)) \quad \text { and } \quad(x(0), y(0))=(a, b) .
$$

Now

$$
\begin{gathered}
y^{\prime \prime}(t)=\partial_{x}(f)(x(t), y(t))+y^{\prime}(t) \partial_{y}(f)(x(t), y(t))=0 \\
7
\end{gathered}
$$


and hence, the flow of $X_{f}$ is given by $\phi(t,(x, y))=(t+x, f(x, y) t+y)$. The relation $\phi(t+s,(x, y))=$ $\phi(t, \phi(s,(x, y)))$ implies that the map $F(x, y)=(1, f(x, y))$ satisfies

$$
F(u+t F(u))=F(u), u \in \mathbb{R}^{2}, t \in \mathbb{R} .
$$

Let $u, v \in \mathbb{R}^{2}$ such that $F(u)$ and $F(v)$ are linearly independent. Then there exists $s, t \in \mathbb{R}$ such that $u-v=t F(u)+s F(v)$ and hence $F(u)=F(v)$ which is a contradiction. So $F(x, y)=\alpha(x, y)(a, b)$, i.e., $(1, f(x, y))=(\alpha(x, y) a, \alpha(x, y) b)$ and $\alpha$ must be constant and hence $f$ is constant.

\section{Proof of Theorem 2.11}

Proof. Let $\left(x_{1}, \ldots, x_{n}\right)$ be an affine coordinates system near $m$ such that $\left(X_{1}, \ldots, X_{n-1}\right)$ are linearly independent in a neighborhood of $m$, where $X_{i}=h_{\#}\left(d x_{i}\right), X_{n}=\sum_{j=1}^{n-1} f_{j} X_{j}$ and, by virtue of the proof of Theorem 2.4 for any $1 \leq i<j \leq n,\left[X_{i}, X_{j}\right]=0$. For any $i=1, \ldots, n-1$, the relation $\left[X_{i}, X_{n}\right]=0$ is equivalent to

$$
X_{i}\left(f_{j}\right)=h_{i n} \partial_{x_{n}}\left(f_{j}\right)+\sum_{l=1}^{n-1} h_{i l} \partial_{x_{l}}\left(f_{j}\right)=0, \quad j=1, \ldots, n-1 .
$$

But $h_{i n}=X_{n}\left(x_{i}\right)=\sum_{l=1}^{n-1} f_{l} h_{i l}$ and hence, for any $i, j=1, \ldots n-1$,

$$
\sum_{l=1}^{n-1} h_{i l}\left(f_{l} \partial_{x_{n}}\left(f_{j}\right)+\partial_{x_{l}}\left(f_{j}\right)\right)=0
$$

Or the matrix $\left(h_{i j}\right)_{1 \leq i, j \leq n-1}$ is invertible so we get

$$
f_{l} \partial_{x_{n}}\left(f_{j}\right)+\partial_{x_{l}}\left(f_{j}\right)=0, \quad l, j=1, \ldots, n-1 .
$$

For $l=j$ we get that $f_{j}$ satisfies $f_{j} \partial_{x_{n}}\left(f_{j}\right)+\partial_{x_{j}}\left(f_{j}\right)=0$ so, according to Lemma 2.13, $\partial_{x_{n}}\left(f_{j}\right)=$ $\partial_{x_{j}}\left(f_{j}\right)=0$ and, from (2.5), $f_{j}=$ constant. We consider $y=f_{1} x_{1}+\ldots+f_{n-1} x_{n-1}-x_{n}$, we have $h_{\#}(d y)=0$ and $\left(x_{1}, \ldots, x_{n-1}, y\right)$ is an affine coordinates system around $m$.

On the other hand, there exists a coordinates system $\left(z_{1}, \ldots, z_{n}\right)$ such that

$$
h_{\sharp}\left(d x_{i}\right)=\partial_{z_{i}}, i=1, \ldots, n-1 .
$$

We deduce that

$$
\partial_{x_{i}}=\sum_{j=1}^{n-1} h^{i j} \partial_{z_{j}}, i=1, \ldots, n-1,
$$

with $h^{i j}=\frac{\partial z_{j}}{\partial x_{i}}$. We consider $\sigma=\sum_{j=1}^{n-1} z_{j} d x_{j}$. We have $d \sigma=0$ so according to the foliated Poincaré Lemma (see[6, p.56]) there exists a function $f$ such that $h^{i j}=\frac{\partial^{2} f}{\partial x_{i} \partial x_{j}}$. 


\subsection{The divergence and the modular class of a contravariant pseudo-Hessian manifold}

We define now the divergence of a contravariant pseudo-Hessian structure. We recall first the definition of the divergence of multivector fields associated to a connection on a manifold.

Let $(M, \nabla)$ be a manifold endowed with a connection. We define $\operatorname{div}_{\nabla}: \Gamma\left(\otimes^{p} T M\right) \rightarrow$ $\Gamma\left(\otimes^{p-1} T M\right)$ by

$$
\operatorname{div}_{\nabla}(T)\left(\alpha_{1}, \ldots, \alpha_{p-1}\right)=\sum_{i=1}^{n} \nabla_{e_{i}}(T)\left(e_{i}^{*}, \alpha_{1}, \ldots, \alpha_{p-1}\right),
$$

where $\alpha_{1}, \ldots, \alpha_{p-1} \in T_{x}^{*} M,\left(e_{1}, \ldots, e_{n}\right)$ a basis of $T_{x} M$ and $\left(e_{1}^{*}, \ldots, e_{n}^{*}\right)$ its dual basis. This operator respects the symmetries of tensor fields.

Suppose now that $(M, \nabla, h)$ is a contravariant pseudo-Hessian manifold. The divergence of this structure is the vector field $\operatorname{div}_{\nabla}(h)$. This vector field is an invariant of the pseudo-Hessian structure and has an important property. Indeed, let $\mathbf{d}_{h}: \Gamma\left(\wedge^{\bullet} T M\right) \longrightarrow \Gamma\left(\wedge^{\bullet+1} T M\right)$ be the differential associated to the Lie algebroid structure $\left(T^{*} M, h_{\#},[,]_{h}\right)$ and given by

$$
\begin{aligned}
\mathbf{d}_{h} Q\left(\alpha_{1}, \ldots, \alpha_{p}\right)= & \sum_{j=1}^{p}(-1)^{j+1} h_{\#}\left(\alpha_{j}\right) \cdot Q\left(\alpha_{1}, \ldots, \hat{\alpha_{j}}, \ldots \alpha_{p}\right) \\
& +\sum_{1 \leq i<j \leq p}(-1)^{i+j} Q\left(\left[\alpha_{i}, \alpha_{j}\right]_{h}, \alpha_{1}, \ldots, \hat{\alpha}_{i}, \ldots, \hat{\alpha_{j}}, \ldots, \alpha_{p}\right) .
\end{aligned}
$$

Proposition 2.14. $\mathbf{d}_{h}\left(\operatorname{div}_{\nabla}(h)\right)=0$.

Proof. Let $\left(x_{1}, \ldots, x_{n}\right)$ be an affine coordinates system. We have

$$
\begin{aligned}
\mathbf{d}_{h} \operatorname{div}_{\nabla}(h)(\alpha, \beta) & =\sum_{i=1}^{n}\left(h_{\#}(\alpha) \cdot \nabla_{\partial_{x_{i}}}(h)\left(d x_{i}, \beta\right)-h_{\#}(\beta) \cdot \nabla_{\partial_{x_{i}}}(h)\left(d x_{i}, \alpha\right)-\nabla_{\partial_{x_{i}}}(h)\left(d x_{i}, \nabla_{h_{\sharp}(\alpha)} \beta\right)+\nabla_{\partial_{x_{i}}}(h)\left(d x_{i}, \nabla_{h_{\sharp}(\beta)} \alpha\right)\right) \\
& =\sum_{i=1}^{n}\left(\nabla_{h_{\sharp}(\alpha)} \nabla_{\partial_{x_{i}}}(h)\left(d x_{i}, \beta\right)-\nabla_{h_{\sharp}(\beta)} \nabla_{\partial_{x_{i}}}(h)\left(d x_{i}, \alpha\right)\right) \\
& \stackrel{\text { 2.1] }}{=} \sum_{i=1}^{n}\left(\nabla_{\left[h_{\sharp}(\alpha), \partial_{x_{i}}\right]}(h)\left(d x_{i}, \beta\right)-\nabla_{\left[h_{\#}(\beta), \partial_{x_{i}}\right]}(h)\left(d x_{i}, \alpha\right)\right) .
\end{aligned}
$$

If we take $\alpha=d x_{l}$ and $\beta=d x_{k}$, we have

$$
\left[\partial_{x_{i}}, h_{\#}\left(d x_{l}\right)\right]=\sum_{m=1}^{n} \partial_{x_{i}}\left(h_{m l}\right) \partial_{x_{m}}
$$

and hence

$$
\mathbf{d}_{h} \operatorname{div}_{\nabla}(h)(\alpha, \beta)=\sum_{i, m=1}^{n}\left(\partial_{x_{i}}\left(h_{m l}\right) \partial_{x_{m}}\left(h_{i k}\right)-\partial_{x_{i}}\left(h_{m k}\right) \partial_{x_{m}}\left(h_{i l}\right)\right)=0 .
$$

Let $(M, \nabla, h)$ be an orientable contravariant pseudo-Hessian manifold and $\Omega$ a volume form on $M$. For any $f$ we denote by $X_{f}=h_{\#}(d f)$ and we define $\mathbf{M}_{\Omega}: C^{\infty}(M, \mathbb{R}) \longrightarrow C^{\infty}(M, \mathbb{R})$ by putting for any $f \in C^{\infty}(M, \mathbb{R})$,

$$
\nabla_{X_{f}} \Omega=\underset{9}{\mathbf{M}_{\Omega}}(f) \Omega .
$$


It is obvious that $\mathbf{M}_{\Omega}$ is a derivation and hence a vector field and $\mathbf{M}_{e^{f} \Omega}=X_{f}+\mathbf{M}_{\Omega}$. Moreover, if $\left(x_{1}, \ldots, x_{n}\right)$ is an affine coordinates system and $\mu=\Omega\left(\partial_{x_{1}}, \ldots, \partial_{x_{n}}\right)$ then

$$
\nabla_{X_{f}} \Omega\left(\partial_{x_{1}}, \ldots, \partial_{x_{n}}\right)=X_{f}(\mu)=X_{\ln |\mu|}(f) \mu .
$$

So in the coordinates system $\left(x_{1}, \ldots, x_{n}\right)$, we have $\mathbf{M}_{\Omega}=X_{\ln |\mu|}$. This implies $\mathbf{d}_{h} \mathbf{M}_{\Omega}=0$. The cohomology class of $\mathbf{M}_{\Omega}$ doesn't depend on $\Omega$ and we call it the modular class of $(M, \nabla, h)$.

Proposition 2.15. The modular class of $(M, \nabla, h)$ vanishes if and only if there exists a volume form $\Omega$ such that $\nabla_{X_{f}} \Omega=0$ for any $f \in C^{\infty}(M, \mathbb{R})$.

By analogy with the case of Poisson manifolds, one can ask if it is possible to find a volume form $\Omega$ such that $\mathcal{L}_{X_{f}} \Omega=0$ for any $f \in C^{\infty}(M, \mathbb{R})$. The following proposition gives a negative answer to this question unless $h=0$.

Proposition 2.16. Let $(M, \nabla, h)$ be an orientable contravariant pseudo-Hessian manifold. Then:

1. For any volume form $\Omega$ and any $f \in C^{\infty}(M, \mathbb{R})$,

$$
\mathcal{L}_{X_{f}} \Omega=\left[\mathbf{M}_{\Omega}(f)+\operatorname{div}_{\nabla}(h)(f)+<h, \operatorname{Hess}(f)>\right] \Omega,
$$

where $\operatorname{Hess}(f)(X, Y)=\nabla_{X}(d f)(Y)$ and $<h$, Hess $(f)>$ is the pairing between the bivector field $h$ and the 2-form $\operatorname{Hess}(f)$.

2. There exists a volume form $\Omega$ such that $\mathcal{L}_{X_{f}} \Omega=0$ for any $f \in C^{\infty}(M, \mathbb{R})$ if and only if $h=0$.

Proof. 1. Let $\left(x_{1}, \ldots, x_{n}\right)$ be an affine coordinates system. Then:

$$
\begin{aligned}
{\left[X_{f}, \partial_{x_{i}}\right] } & =\sum_{l, j=1}^{n}\left[\partial_{x_{j}}(f) h_{j l} \partial_{x_{l}}, \partial_{x_{i}}\right] \\
& =-\sum_{l, j=1}^{n}\left(h_{j l} \partial_{x_{i}} \partial_{x_{j}}(f)+\partial_{x_{j}}(f) \partial_{x_{i}}\left(h_{j l}\right)\right) \partial_{x_{l}} \\
\mathcal{L}_{X_{f}} \Omega\left(\partial_{x_{1}}, \ldots, \partial_{x_{n}}\right) & =\left(\nabla_{X_{f}} \Omega\right)\left(\partial_{x_{1}}, \ldots, \partial_{x_{n}}\right)-\sum_{i=1}^{n} \Omega\left(\left(\partial_{x_{1}}, \ldots,\left[X_{f}, \partial_{x_{i}}\right], \ldots, \partial_{x_{n}}\right)\right) \\
& =\left(\nabla_{X_{f}} \Omega\right)\left(\partial_{x_{1}}, \ldots, \partial_{x_{n}}\right)+\sum_{i, j=1}^{n}\left(h_{j i} \partial_{x_{i}} \partial_{x_{j}}(f)+\partial_{x_{j}}(f) \partial_{x_{i}}\left(h_{j i}\right)\right) \Omega\left(\partial_{x_{1}}, \ldots, \partial_{x_{n}}\right)
\end{aligned}
$$

and the formula follows since $\operatorname{div}_{\nabla}(h)=\sum_{i, j=1}^{n} \partial_{x_{i}}\left(h_{j i}\right) \partial_{x_{j}}$.

2. This is a consequence of the fact that $\mathbf{M}_{\Omega}$ and $\operatorname{div}_{\nabla}(h)$ are derivation and

$$
<h, \operatorname{Hess}(f g)>=f<h, \operatorname{Hess}(g)>+g<h, \operatorname{Hess}(f)>+<h, d f \odot d g>.
$$




\section{The tangent bundle of a contravariant pseudo-Hessian manifold}

In this section, we define and study the associated Poisson tensor on the tangent bundle of a contravariant pseudo-Hessian manifold. One can see [7] for the classical properties of the tangent bundle of a manifold endowed with a linear connection.

Let $(M, \nabla)$ be an affine manifold, $p: T M \longrightarrow M$ the canonical projection and $K: T T M \longrightarrow$ $T M$ the connection map of $\nabla$ locally given by

$$
K\left(\sum_{i=1}^{n} b_{i} \partial_{x_{i}}+\sum_{j=1}^{n} Z_{j} \partial_{\mu_{j}}\right)=\sum_{l=1}^{n}\left(Z_{l}+\sum_{i=1}^{n} \sum_{j=1}^{n} b_{i} \mu_{j} \Gamma_{i j}^{l}\right) \partial_{x_{l}},
$$

where $\left(x_{1}, \ldots, x_{n}\right)$ is a system of local coordinates, $\left(x_{1}, \ldots, x_{n}, \mu_{1}, \ldots, \mu_{n}\right)$ the associated system of coordinates on $T M$ and $\nabla_{\partial_{x_{i}}} \partial_{x_{j}}=\sum_{l=1}^{n} \Gamma_{i j}^{l} \partial_{x_{l}}$. Then

$$
T T M=\operatorname{ker} T p \oplus \operatorname{ker} K .
$$

For $X \in \Gamma(T M)$, we denote by $X^{h}$ its horizontal lift and by $X^{v}$ its vertical lift. The flow of $X^{v}$ is given by $\Phi^{X}(t,(x, u))=(x, u+t X(x))$ and $X^{h}(x, u)=h^{(x, u)}(X(x))$, where $h^{(x, u)}: T_{x} M \longrightarrow$ $\operatorname{ker} K(x, u)$ is the inverse of the restriction of $d p$ to $\operatorname{ker} K(x, u)$. Since the curvature of $\nabla$ vanishes, we have

$$
\left[X^{h}, Y^{h}\right]=[X, Y]^{h},\left[X^{h}, Y^{v}\right]=\left(\nabla_{X} Y\right)^{v} \quad \text { and } \quad\left[X^{v}, Y^{v}\right]=0,
$$

for any $X, Y \in \Gamma(T M)$. For any $\alpha \in \Omega^{1}(M)$, we define $\alpha^{v}, \alpha^{h} \in \Omega^{1}(T M)$ by

$$
\left\{\begin{array} { l } 
{ \alpha ^ { v } ( X ^ { v } ) = \alpha ( X ) \circ p , } \\
{ \alpha ^ { v } ( X ^ { h } ) = 0 , }
\end{array} \quad \text { and } \quad \left\{\begin{array}{l}
\alpha^{h}\left(X^{h}\right)=\alpha(X) \circ p, \\
\alpha^{h}\left(X^{v}\right)=0 .
\end{array}\right.\right.
$$

The following proposition is well-known [7] and can be proved easily.

Proposition 3.1. The connection $\bar{\nabla}$ on $T M$ given by

$$
\bar{\nabla}_{X^{h}} Y^{h}=\left(\nabla_{X} Y\right)^{h}, \bar{\nabla}_{X^{h}} Y^{v}=\left(\nabla_{X} Y\right)^{v} \quad \text { and } \quad \bar{\nabla}_{X^{v}} Y^{h}=\bar{\nabla}_{X^{v}} Y^{v}=0
$$

where $X, Y \in \Gamma(T M)$, defines an affine structure on TM. Moreover, the endomorphism vector field $J: T T M \longrightarrow T T M$ given by $J X^{h}=X^{v}$ and $J X^{v}=-X^{h}$ satisfies $J^{2}=-\operatorname{Id}_{T T M}$, is parallel with respect to $\bar{\nabla}$ and hence defines a complex structure on $T M$.

Let $h$ be a symmetric bivector field on $M$. We associate to $h$ a skew-symmetric bivector field $\Pi$ on $T M$ by putting

$$
\Pi\left(\alpha^{v}, \beta^{v}\right)=\Pi\left(\alpha^{h}, \beta^{h}\right)=0 \quad \text { and } \quad \Pi\left(\alpha^{h}, \beta^{v}\right)=-\Pi\left(\beta^{v}, \alpha^{h}\right)=h(\alpha, \beta) \circ p,
$$

for any $\alpha, \beta \in \Omega^{1}(M)$. For any $\alpha \in \Omega^{1}(M)$,

$$
\Pi_{\#}\left(\alpha^{v}\right)=-h_{\#}(\alpha)^{h} \quad \text { and } \quad \Pi_{\#}\left(\alpha^{h}\right)=h_{\#}(\alpha)^{v} .
$$

To prove one of our main result in this section, we need the following proposition which is a part of the folklore. 
Proposition 3.2. Let $(P, \nabla)$ be a manifold endowed with a torsionless connection and $\pi$ is a bivector field on $P$. Then the Nijenhuis-Schouten bracket $[\pi, \pi]$ is given by

$$
[\pi, \pi](\alpha, \beta, \gamma)=2\left(\nabla_{\pi_{\#}(\alpha)} \pi(\beta, \gamma)+\nabla_{\pi_{\#}(\beta)} \pi(\gamma, \alpha)+\nabla_{\pi_{\#}(\gamma)} \pi(\alpha, \beta)\right) .
$$

Theorem 3.3. The following assertions are equivalent:

(i) $(M, \nabla, h)$ is a contravariant pseudo-Hessian manifold.

(ii) $(T M, \Pi)$ is a Poisson manifold.

In this case, if $L$ is a leaf of $\operatorname{Im} h_{\#}$ then $T L \subset T M$ is a symplectic leaf of $\Pi$ which is also a complex submanifold of TM. Moreover, if $\omega_{L}$ is the symplectic form of TL induced by $\Pi$ and $g_{L}$ is the pseudo-Riemannian metric given by $g_{L}(U, V)=\omega(J U, V)$ then $\left(T L, g_{L}, \omega_{L}, J\right)$ is a pseudo-Kähler manifold.

Proof. We will use Proposition 3.2 to prove the equivalence. Indeed, by a direct computation one can establish easily, for any $\alpha, \beta, \gamma \in \Omega^{1}(M)$, the following relations

$$
\begin{aligned}
& \bar{\nabla}_{\Pi_{\#}\left(\alpha^{v}\right)} \Pi\left(\beta^{v}, \gamma^{v}\right)=\bar{\nabla}_{\Pi_{\#}\left(\alpha^{v}\right)} \Pi\left(\beta^{h}, \gamma^{h}\right)=\bar{\nabla}_{\Pi_{\#}\left(\alpha^{h}\right)} \Pi\left(\beta^{v}, \gamma^{v}\right)=\bar{\nabla}_{\Pi_{\#}\left(\alpha^{h}\right)} \Pi\left(\beta^{h}, \gamma^{h}\right)=\bar{\nabla}_{\Pi_{\#}\left(\alpha^{h}\right)} \Pi\left(\beta^{h}, \gamma^{v}\right)=0, \\
& \bar{\nabla}_{\Pi_{\#}\left(\alpha^{v}\right)} \Pi\left(\beta^{h}, \gamma^{v}\right)=\nabla_{h_{\#}(\alpha)}(h)(\beta, \gamma) \circ p,
\end{aligned}
$$

and the equivalence follows. The second part of the theorem is obvious and the only point which need to be checked is that $g_{L}$ is nondegenerate.

Remark 3.4. 1. The total space of the dual of a Lie algebroid carries a Poisson tensor (see [12]). If $(M, \nabla, h)$ is a contravariant pseudo-Hessian manifold then, according to Theorem 2.4. $T^{*} M$ carries a Lie algebroid structure and one can see easily that $\Pi$ is the corresponding Poisson tensor on TM.

2. The equivalence of (i) and (ii) in Theorem 3.3 deserves to be stated explicitly in the case of $\mathbb{R}^{n}$ endowed with its canonical affine structure $\nabla$. Indeed, let $\left(h_{i j}\right)_{1 \leq i, j \leq n}$ be a symmetric matrix where $h_{i j} \in C^{\infty}\left(\mathbb{R}^{n}, \mathbb{R}\right)$ and $h$ the associated symmetric bivector field on $\mathbb{R}^{n}$. The associated bivector field $\Pi_{h}$ on $T \mathbb{R}^{n}=\mathbb{C}^{n}$ is

$$
\Pi_{h}=\sum_{i, j=1}^{n} h_{i j}(x) \partial_{x_{i}} \wedge \partial_{y_{j}},
$$

where $\left(x_{1}+i y_{1}, \ldots, x_{n}+i y_{n}\right)$ are the canonical coordinates of $\mathbb{C}^{n}$. Then, according to Theorem $2.4\left(\mathbb{R}^{n}, \nabla, h\right)$ is a contravariant pseudo-Hessian manifold if and only if $\left(\mathbb{C}^{n}, \Pi_{h}\right)$ is a Poisson manifold.

We explore now some relations between some invariants of $(M, \nabla, h)$ and some invariants of $(T M, \Pi)$.

Proposition 3.5. Let $(M, \nabla, h)$ be a contravariant pseudo-Hessian manifold. Then $\left(\operatorname{div}_{\nabla} h\right)^{v}=$ $\operatorname{div}_{\bar{\nabla}} \Pi$.

Proof. Fix $(x, u) \in T M$ and choose a basis $\left(e_{1}, \ldots, e_{n}\right)$ of $T_{x} M$. Then $\left(e_{1}^{v}, \ldots, e_{n}^{v}, e_{1}^{h}, \ldots, e_{n}^{h}\right)$ is a basis of $T_{(x, u)} T M$ with $\left(\left(e_{1}^{*}\right)^{v}, \ldots,\left(e_{n}^{*}\right)^{v},\left(e_{1}^{*}\right)^{h}, \ldots,\left(e_{n}^{*}\right)^{h}\right)$ as a dual basis. For any $\alpha \in T_{x}^{*} M$, we have

$$
\begin{aligned}
\prec \alpha^{v}, \operatorname{div}_{\bar{\nabla}} \Pi> & =\sum_{i=1}^{n}\left(\bar{\nabla}_{e_{i}^{v}}(\Pi)\left(\left(e_{i}^{*}\right)^{v}, \alpha^{v}\right)+\bar{\nabla}_{e_{i}^{h}}(\Pi)\left(\left(e_{i}^{*}\right)^{h}, \alpha^{v}\right)\right) \\
& \stackrel{3.2}{=} \quad<\alpha, \operatorname{div}_{\nabla}(h)>\circ p=<\alpha^{v},\left(\operatorname{div}_{\nabla}(h)\right)^{v}>.
\end{aligned}
$$


In the same way we get that $\left\langle\alpha^{h}, \operatorname{div}_{\bar{\nabla}} \Pi>=0\right.$ and the result follows.

Let $(M, \nabla, h)$ be a contravariant pseudo-Hessian manifold. For any multivector field $Q$ on $M$ we define its vertical lift $Q^{v}$ on $T M$ by

$$
i_{\alpha^{h}} Q^{v}=0 \quad \text { and } Q^{v}\left(\alpha_{1}^{v}, \ldots, \alpha_{q}^{v}\right)=Q\left(\alpha_{1}, \ldots, \alpha_{q}\right) \circ p .
$$

Recall that $h$ defines a Lie algebroid structure on $T^{*} M$ whose anchor is $h_{\#}$ and the Lie bracket is given by (2.3). The Poisson tensor $\Pi$ defines a Lie algebroid structure on $T^{*} T M$ whose anchor is $\Pi_{\#}$ and the Lie bracket is the Koszul bracket

$$
\left[\phi_{1}, \phi_{2}\right]_{\Pi}=\mathcal{L}_{\Pi_{\#(}\left(\phi_{1}\right)} \phi_{2}-\mathcal{L}_{\Pi_{\#(t}\left(\phi_{2}\right)} \phi_{1}-d \Pi\left(\phi_{1}, \phi_{2}\right), \quad \phi_{1}, \phi_{2} \in \Omega^{1}(T M) .
$$

We denote by $\mathbf{d}_{h}$ (resp. $\mathbf{d}_{\Pi}$ ) the differential associated to the Lie algebroid structure on $T^{*} M$ (resp. $T^{*} T M$ ) defined by $h$ (resp. $\Pi$ ).

Proposition 3.6. (i) For any $\alpha, \beta \in \Omega^{1}(M)$ and $X \in \Gamma(T M)$,

$$
\left\{\begin{array}{l}
\mathcal{L}_{X^{h}} \alpha^{h}=\left(\mathcal{L}_{X} \alpha\right)^{h}, \mathcal{L}_{X^{h}} \alpha^{v}=\left(\nabla_{X} \alpha\right)^{v}, \mathcal{L}_{X^{v}} \alpha^{h}=0 \quad \text { and } \mathcal{L}_{X^{v}} \alpha^{v}=\left(\mathcal{L}_{X} \alpha\right)^{h}-\left(\nabla_{X} \alpha\right)^{h}, \\
{\left[\alpha^{h}, \beta^{h}\right]_{\Pi}=0,\left[\alpha^{v}, \beta^{v}\right]_{\Pi}=-[\alpha, \beta]_{h}^{v} \text { and }\left[\alpha^{h}, \beta^{v}\right]_{\Pi}=\left(\mathcal{D}_{\beta} \alpha\right)^{h},}
\end{array}\right.
$$

where $\mathcal{D}$ is the connection given by (2.4).

(ii) $\left(\mathbf{d}_{h} Q\right)^{v}=-\mathbf{d}_{\Pi}\left(Q^{v}\right)$.

Proof. The relations in (i) can be established by a straightforward computation. From these relations and the fact that $\Pi_{\#}\left(\alpha^{h}\right)=\left(h_{\#}(\alpha)\right)^{v}$ one can deduce easily that $i_{\alpha^{h}} \mathbf{d}_{\Pi}\left(Q^{v}\right)=0$. On the other hand, since $\Pi_{\#}\left(\alpha^{v}\right)=-\left(h_{\#}(\alpha)\right)^{h}$ and $\left[\alpha^{v}, \beta^{v}\right]_{\Pi}=-[\alpha, \beta]^{v}$ we can conclude.

Remark 3.7. From Propositions 2.14 and Proposition 3.6 we can deduce that $\mathbf{d}_{\Pi}\left(\operatorname{div}_{\bar{\nabla}} \Pi\right)=0$. This is not a surprising result because $\bar{\nabla}$ is flat and $\operatorname{div}_{\bar{\nabla}} \Pi$ is a representative of the modular class of $\Pi$.

As a consequence of Proposition 3.6 we can define a linear map from the cohomology of $\left(T^{*} M, h_{\#},[,]_{h}\right)$ to the cohomology of $\left(T^{*} T M, \Pi_{\#},[,]_{\Pi}\right)$ by

$$
V: H^{*}(M, h) \longrightarrow H^{*}(T M, \Pi),[Q] \mapsto\left[Q^{v}\right]
$$

Proposition 3.8. $V$ is injective.

Proof. An element $P \in \Gamma\left(\wedge^{d} T T M\right)$ is of type $(r, d-r)$ if for any $q \neq r$

$$
P\left(\alpha_{1}^{v}, \ldots, \alpha_{q}^{v}, \beta_{1}^{h}, \ldots, \beta_{d-q}^{h}\right)=0,
$$

for any $\alpha_{1}, \ldots, \alpha_{q}, \beta_{1}, \ldots, \beta_{d-q} \in \Omega^{1}(M)$. We have

$$
\left\{\begin{array}{l}
\Gamma\left(\wedge^{d} T T M\right)=\bigoplus_{r=0}^{d} \Gamma_{(r, d-r)}\left(\wedge^{d} T T M\right) \\
\mathbf{d}_{\Pi}\left(\Gamma_{(r, d-r)}\left(\wedge^{d} T T M\right)\right) \subset \Gamma_{(r+1, d-r)}\left(\wedge^{d+1} T T M\right) \oplus \Gamma_{(r, d+1-r)}\left(\wedge^{d+1} T T M\right) .
\end{array}\right.
$$

Let $Q \in \Gamma\left(\wedge^{d} T M\right)$ such that $\mathbf{d}_{h} Q=0$ and there exists $P \in \Gamma\left(\wedge^{d-1} T T M\right)$ such that $\mathbf{d}_{\Pi} P=$ $Q^{v}$. Since $Q^{v} \in \Gamma_{(d, 0)}\left(\wedge^{d} T T M\right)$ then $P \in \Gamma_{(d-1,0)}\left(\wedge^{d-1} T T M\right)$. Let us show that $P=T^{v}$. For $\alpha_{1}, \ldots, \alpha_{d-1}, \beta \in \Omega^{1}(M)$, we have

$$
0=\mathbf{d}_{\Pi} P\left(\beta^{h}, \alpha_{1}^{v}, \ldots, \alpha_{d-1}^{v}\right)=\left(h_{\#}(\beta)\right)^{v} . P\left(\alpha_{1}^{v}, \ldots, \alpha_{d-1}^{v}\right) .
$$

So the function $P\left(\alpha_{1}^{v}, \ldots, \alpha_{d-1}^{v}\right)$ is constant on the fibers of $T M$ and hence there exists $T \in$ $\Gamma\left(\wedge^{d-1} T M\right)$ such that $P\left(\alpha_{1}^{v}, \ldots, \alpha_{d-1}^{v}\right)=T\left(\alpha_{1}, \ldots, \alpha_{d-1}\right) \circ p$. So $[Q]=0$ which completes the proof. 


\section{Linear, affine and multiplicative contravariant pseudo-Hessian structures}

\subsection{Linear and affine contravariant pseudo-Hessian structures}

As in the Poisson geometry context, we have the notions of linear and affine contravariant pseudo-Hessian structures. One can see [11] for the notion of cocycle in associative algebras.

Let $(V, \nabla)$ be a finite dimensional real vector space endowed with its canonical affine structure. A symmetric bivector field $h$ on $V$ is called affine if there exists a commutative product $\bullet$ on $V^{*}$ and a symmetric bilinear form $B$ on $V^{*}$ such that, for any $\alpha, \beta \in V^{*} \subset \Omega^{1}(V)$ and $u \in V$,

$$
h(\alpha, \beta)(u)=<\alpha \bullet \beta, u>+B(\alpha, \beta) .
$$

One can see easily that if $\alpha, \beta \in \Omega^{1}(V)=C^{\infty}\left(V, V^{*}\right)$ then

$$
h(\alpha, \beta)(u)=<\alpha(u) \bullet \beta(u), u>+B(\alpha(u), \beta(u)) .
$$

If $B=0, h$ is called linear.

If $\left(x_{1}, \ldots, x_{n}\right)$ is a linear coordinates system on $V^{*}$ associated to a basis $\left(e_{1}, \ldots, e_{n}\right)$ then

$$
h\left(d x_{i}, d x_{j}\right)=b_{i j}+\sum_{k=1}^{n} C_{i j}^{k} x_{k},
$$

where $e_{i} \bullet e_{j}=\sum_{k=1}^{n} C_{i j}^{k} e_{k}$ and $b_{i j}=B\left(e_{i}, e_{j}\right)$.

Proposition 4.1. $(V, \nabla, h)$ is a contravariant pseudo-Hessian manifold if and only if $\bullet$ is associative and $B$ is a scalar 2-cocycle of $\left(V^{*}, \bullet\right)$, i.e.,

$$
B(\alpha \bullet \beta, \gamma)=B(\alpha, \beta \bullet \gamma)
$$

for any $\alpha, \beta, \gamma \in V^{*}$.

Proof. For any $\alpha \in V^{*}$ and $u \in V, h_{\#}(\alpha)(u)=\mathrm{L}_{\alpha}^{*} u+i_{\alpha} B$ where $\mathrm{L}_{\alpha}(\beta)=\alpha \bullet \beta$ and $i_{\alpha} B \in V^{* *}=V$. We denote by $\phi^{h_{\#}(\alpha)}$ the flow of the vector field $h_{\#}(\alpha)$. Then, for any $\alpha, \beta, \gamma \in V^{*}$,

$$
\begin{aligned}
\nabla_{h_{\#}(\alpha)}(h)(\beta, \gamma)(u) & =\frac{d}{d t}_{\mid t=0}\left(<\beta \bullet \gamma, \phi^{h_{\#}(\alpha)}(t, u)>+B(\beta, \gamma)\right) \\
& =<\beta \bullet \gamma, \mathrm{L}_{\alpha}^{*} u+i_{\alpha} B> \\
& =<\alpha \bullet(\beta \bullet \gamma), u>+B(\alpha, \beta \bullet \gamma)
\end{aligned}
$$

and the result follows.

Conversely, we have the following result.

Proposition 4.2. Let $(\mathcal{A}, \bullet, B)$ be a commutative and associative algebra endowed with a symmetric scalar 2-cocycle. Then:

1. $\mathcal{A}^{*}$ carries a structure of a contravariant pseudo-Hessian structure $(\nabla, h)$ where $\nabla$ is the canonical affine structure of $\mathcal{A}^{*}$ and $h$ is given by

$$
h(u, v)(\alpha)=<\alpha, u(\alpha) \bullet v(\alpha)>+B(u(\alpha), v(\alpha)), \quad \alpha \in \mathcal{A}^{*}, u, v \in \Omega^{1}\left(\mathcal{A}^{*}\right) .
$$


2. When $B=0$, the leaves of the affine foliation associated to $\operatorname{Im} h_{\#}$ are the orbits of the action $\Phi$ of $(\mathcal{A},+)$ on $\mathcal{A}^{*}$ given by $\Phi(u, \alpha)=\exp \left(\mathrm{L}_{u}^{*}\right)(\alpha)$

3. The associated Poisson tensor $\Pi$ on $T \mathcal{A}^{*}=\mathcal{A}^{*} \times \mathcal{A}^{*}$ is the affine Poisson tensor dual associated to the Lie algebra $(\mathcal{A} \times \mathcal{A},[]$,$) endowed with the 2-cocycle B_{0}$ where

$$
[(a, b),(c, d)]=(a \bullet d-b \bullet c, 0) \quad \text { and } \quad B_{0}((a, b),(c, d))=B(a, d)-B(c, b) .
$$

Proof. It is only the third point which need to be checked. One can see easily that [, ] is a Lie bracket on $\mathcal{A} \times \mathcal{A}$ and $B_{0}$ is a scalar 2-cocycle for this Lie bracket. For any $a \in \mathcal{A} \subset \Omega^{1}\left(\mathcal{A}^{*}\right)$, $a^{v}=(0, a) \in \mathcal{A} \times \mathcal{A} \subset \Omega^{1}\left(\mathcal{A}^{*} \times \mathcal{A}^{*}\right)$ and $a^{h}=(a, 0)$. So

$$
\Pi\left(a^{h}, b^{v}\right)(\alpha, \beta)=h(a, b)(\alpha)=<\alpha, a \bullet b>+B(a, b) .
$$

On the the other hand, if $\Pi^{*}$ is the Poisson tensor dual, then

$$
\begin{aligned}
\Pi^{*}\left(a^{h}, b^{v}\right)(\alpha, \beta) & =\Pi^{*}((a, 0),(0, b))(\alpha, \beta) \\
& =<(\alpha, \beta),[(a, 0),(0, b)]>+B_{0}((a, 0),(0, b)) \\
& =<\alpha, a \bullet b>+B(a, b) \\
& =\Pi\left(a^{h}, b^{v}\right)(\alpha, \beta) .
\end{aligned}
$$

In the same way one can check the others equalities.

This proposition can be used as a machinery to build examples of pseudo-Hessian manifolds. Indeed, by virtue of Proposition 2.5, any orbit $L$ of the action $\Phi$ has an affine structure $\nabla_{L}$ and a pseudo-Riemannian metric $g_{L}$ such that $\left(L, \nabla_{L}, g_{L}\right)$ is a pseudo-Hessian manifold.

Example 4.3. We take $\mathcal{A}=\mathbb{R}^{4}$ with its canonical basis $\left(e_{i}\right)_{i=1}^{4}$ and $\left(e_{i}^{*}\right)_{i=1}^{4}$ is the dual basis. We endow $\mathcal{A}$ with the commutative associative product given by

$$
e_{1} \bullet e_{1}=e_{2}, e_{1} \bullet e_{2}=e_{3}, e_{1} \bullet e_{3}=e_{2} \bullet e_{2}=e_{4},
$$

the others products are zero and we endow $\mathcal{A}^{*}$ with the linear contravariant pseudo-Hessian structure associated to $\bullet$. We denote by $(a, b, c, d)$ the linear coordinates on $\mathcal{A}$ and $(x, y, z, t)$ the dual coordinates on $\mathcal{A}^{*}$. We have

$\Phi\left(a e_{1}+b e_{2}+c e_{3}+d e_{4}^{*}, x e_{1}^{*}+y e_{2}^{*}+z e_{3}^{*}+t e_{4}^{*}\right)=\left(x+a y+\left(\frac{1}{2} a^{2}+b\right) z+\left(\frac{1}{6} a^{3}+a b+c\right) t, y+a z+\left(\frac{1}{2} a^{2}+b\right) t, z+a t, t\right)$ and

$$
X_{e_{1}}=y \partial_{x}+z \partial_{y}+t \partial_{z}, X_{e_{2}}=z \partial_{x}+t \partial_{y}, X_{e_{3}}=t \partial_{x} \text { and } X_{e_{4}}=0 .
$$

Let us describe the pseudo-Hessian structure of the hyperplane $M_{c}=\{t=c, c \neq 0\}$ endowed with the coordinates $(x, y, z)$. We denote by $g_{c}$ the pseudo-Riemannian of $M_{c}$. We have, for instance,

$$
g_{c}\left(X_{e_{1}}, X_{e_{1}}\right)(x, y, z, c)=h\left(e_{1}, e_{1}\right)(x, y, z, c)=<e_{1} \bullet e_{1},(x, y, z, c)>=y .
$$

So, one can see that the matrix of $g_{c}$ in $\left(X_{e_{1}}, X_{e_{2}}, X_{e_{3}}\right)$ is the passage matrix $P$ from $\left(X_{e_{1}}, X_{e_{2}}, X_{e_{3}}\right)$ to $\left(\partial_{x}, \partial_{y}, \partial_{z}\right)$ and hence

$$
g_{c}=\frac{1}{c}\left(2 d x d z+d y^{2}-\frac{2 z}{c} d y d z+\frac{\left(z^{2}-y c\right)}{c^{2}} d z^{2}\right) .
$$

The signature of this metric is $(+,+,-)$ if $c>0$ and $(+,-,-)$ if $c<0$. One can check easily that $g_{c}$ is the restriction of $\nabla d \phi$ to $M_{c}$, where

$$
\phi(x, y, z, t)=\frac{z^{4}}{12 t_{15}^{3}}+\frac{y^{2}}{2 t}-\frac{z^{2} y}{2 t}+\frac{x z}{t} .
$$




\subsection{Multiplicative contravariant pseudo-Hessian structures}

A contravariant pseudo-Hessian structure $(\nabla, h)$ on a Lie group $G$ is called multiplicative if the multiplication $m:(G \times G, \nabla \oplus \nabla, h \oplus h) \longrightarrow(G, \nabla, h)$ is affine and sends $h \oplus h$ to $h$.

Lemma 4.4. Let $G$ be a connected Lie group and $\nabla$ a connection on $G$ such that the multiplication $m:(G \times G, \nabla \oplus \nabla) \longrightarrow(G, \nabla)$ preserves the connections. Then $G$ is abelian and $\nabla$ is bi-invariant.

Proof. We will denote by $\chi^{r}(G)$ (resp. $\chi^{l}(G)$ ) the space of left invariant vector fields (resp. the right invariant vector fields) on $G$. It is clear that for any $X \in \chi^{r}(G)$ and $Y \in \chi^{l}(G)$, the vector field $(X, Y)$ on $G \times G$ is $m$-related to the vector field $X+Y$ on $G$ :

$$
\operatorname{Tm}\left(X_{a}, Y_{b}\right)=X_{a} \cdot b+a \cdot Y_{b}=X_{a b}+Y_{a b}=(X+Y)_{a b}
$$

It follows that for any $X_{1}, X_{2} \in \chi^{r}(G)$ and $Y_{1}, Y_{2} \in \chi^{l}(G)$, the vector field $(\nabla \oplus \nabla)_{\left(X_{1}, Y_{1}\right)}\left(X_{2}, Y_{2}\right)$ is $m$-related to $\nabla_{X_{1}+Y_{1}}\left(X_{2}+Y_{2}\right)$, hence:

$$
\operatorname{Tm}\left(\left(\nabla_{X_{1}} X_{2}\right)_{a},\left(\nabla_{Y_{1}} Y_{2}\right)_{b}\right)=\left(\nabla_{\left(X_{1}+Y_{1}\right)}\left(X_{2}+Y_{2}\right)\right)_{a b}
$$

So we get

$$
\left(\nabla_{X_{1}} X_{2}\right)_{a} \cdot b+a \cdot\left(\nabla_{Y_{1}} Y_{2}\right)_{b}=\left(\nabla_{X_{1}} X_{2}+\nabla_{X_{1}} Y_{2}+\nabla_{Y_{1}} X_{2}+\nabla_{Y_{1}} Y_{2}\right)_{a b}
$$

If we take $Y_{1}=0=Y_{2}$ we obtain that $\nabla$ is right invariant. In the same way we get that $\nabla$ is left invariant. Now, if we return back to the equation 4.1 we obtain that for any $X \in \chi^{r}(G)$ and $Y \in \chi^{l}(G)$ we have $\nabla X=0=\nabla Y$. This implies that any left invariant vector field is also right invariant ; indeed, if $Y=\sum_{i=1}^{n} f_{i} X_{i}$ with $Y \in \chi^{l}(G)$ and $X_{i} \in \chi^{r}(G)$ then $X_{j} f_{i}=0$ for all $i, j=1, \cdot, n$. Hence the adjoint representation is trivial and hence $G$ must be abelian.

At the end of the paper, we give another proof of this Lemma based on parallel transport.

Corollary 4.5. Let $(\nabla, h)$ be multiplicative contravariant pseudo-Hessian structure on a simply connected Lie group $G$. Then $G$ is a vector space, $\nabla$ its canonical affine connection and $h$ is linear.

Example 4.6. Based on the classification of complex associative commutative algebras given in [14], we can give a list of examples of affine contravariant pseudo-Hessian structures up to dimension 4.

1. On $\mathbb{R}^{2}$ :

$$
h_{1}=\left(\begin{array}{cc}
x_{2} & 0 \\
0 & 0
\end{array}\right), h_{2}=\left(\begin{array}{cc}
x_{1} & x_{2} \\
x_{2} & 0
\end{array}\right) \quad \text { and } \quad h_{3}=\left(\begin{array}{cc}
x_{2} & 1 \\
1 & 0
\end{array}\right) \text {. }
$$

2. On $\mathbb{R}^{3}$ :

$$
\begin{aligned}
& h_{1}=\left(\begin{array}{ccc}
a & 0 & x_{2} \\
0 & 0 & 0 \\
x_{2} & 0 & b
\end{array}\right), h_{2}=\left(\begin{array}{ccc}
x_{2} & x_{3} & a \\
x_{3} & a & 0 \\
a & 0 & 0
\end{array}\right), h_{3}=\left(\begin{array}{ccc}
a & 0 & x_{1} \\
0 & 0 & x_{2} \\
x_{1} & x_{2} & x_{3}
\end{array}\right), \\
& h_{4}=\left(\begin{array}{ccc}
x_{2} & 0 & x_{2} \\
0 & 0 & x_{2}+a \\
x_{2} & x_{2}+a & x_{3}
\end{array}\right) \text { and } h_{5}=\left(\begin{array}{ccc}
x_{2} & 0 & x_{1} \\
0 & 0 & x_{2} \\
x_{1} & x_{2} & x_{3}
\end{array}\right) .
\end{aligned}
$$


3. On $\mathbb{R}^{4}$ :

$$
\begin{aligned}
h_{1} & =\left(\begin{array}{cccc}
x_{3} & a & x_{4}+b & 0 \\
a & -x_{4}+c & 0 & 0 \\
x_{4}+b & 0 & 0 & 0 \\
0 & 0 & 0 & 0
\end{array}\right), h_{2}=\left(\begin{array}{cccc}
x_{2} & x_{3} & x_{4} & a \\
x_{3} & x_{4} & a & 0 \\
x_{4} & a & 0 & 0 \\
a & 0 & 0 & 0
\end{array}\right), h_{3}=\left(\begin{array}{cccc}
x_{1} & x_{2} & x_{3} & x_{4} \\
x_{2} & 0 & 0 & 0 \\
x_{3} & 0 & 0 & 0 \\
x_{4} & 0 & 0 & 0
\end{array}\right), \\
h_{4} & =\left(\begin{array}{llll}
x_{1} & x_{2} & x_{3} & x_{4} \\
x_{2} & x_{4} & 0 & 0 \\
x_{3} & 0 & 0 & 0 \\
x_{4} & 0 & 0 & 0
\end{array}\right) \text { and } h_{5}=\left(\begin{array}{cccc}
x_{1} & x_{2} & x_{3} & x_{4} \\
x_{2} & x_{3} & x_{4} & 0 \\
x_{3} & x_{4} & 0 & 0 \\
x_{4} & 0 & 0 & 0
\end{array}\right) .
\end{aligned}
$$

\section{Quadratic contravariant pseudo-Hessian structures}

Let $V$ be a vector space of dimension $n$. Denote by $\nabla$ its canonical affine connection. A symmetric bivector field $h$ on $V$ is quadratic if there exists a basis $\mathbb{B}$ of $V$ such that, for any $i, j=1, \ldots, n$,

$$
h\left(d x_{i}, d x_{j}\right)=\sum_{l, k=1}^{n} a_{l, k}^{i, j} x_{l} x_{k},
$$

where the $a_{k, l}^{i, j}$ are real constants and $\left(x_{1}, \ldots, x_{n}\right)$ are the linear coordinates associated to $\mathbb{B}$.

For any linear endomorphism $A$ on $V$ we denote by $\widetilde{A}$ the associated linear vector field on $V$.

The key point is that if $h$ is a quadratic contravariant pseudo-Hessian bivector field on $V$ then its divergence is a linear vector field, i.e., $\operatorname{div}_{\nabla}(h)=\widetilde{L^{h}}$ where $L^{h}$ is a linear endomorphism of $V$. Moreover, if $F=(A, u)$ is an affine transformation of $V$ then $\operatorname{div}_{\nabla}\left(F_{*} h\right)=\widetilde{A^{-1} L^{h} A}$. So the Jordan form of $L_{h}$ is an invariant of the quadratic contravariant pseudo-Hessian structure. By using Maple we can classify quadratic contravariant pseudo-Hessian structures on $\mathbb{R}^{2}$. The same approach has been used by [9] to classify quadratic Poisson structures on $\mathbb{R}^{4}$. Note that if $h$ is a quadratic contravariant pseudo-Hessian tensor on $\mathbb{R}^{n}$ then its associated Poisson tensor on $\mathbb{C}^{n}$ is also quadratic.

Theorem 5.1. 1. Up to an affine isomorphism, there is two quadratic contravariant pseudoHessian structures on $\mathbb{R}^{2}$ which are divergence free

$$
h_{1}=\left(\begin{array}{cc}
0 & 0 \\
0 & u x^{2}
\end{array}\right) \quad \text { and } \quad h_{2}=\left(\begin{array}{cc}
\frac{r^{2} x^{2}}{c}-2 r x y+c y^{2} & \frac{r^{3} x^{2}}{c^{2}}-\frac{2 r^{2} x y}{c}+r y^{2} \\
\frac{r^{3} x^{2}}{c^{2}}-\frac{2 r^{2} x y}{c}+r y^{2} & -\frac{2 r^{3} x y}{c^{2}}+\frac{r^{4} x^{2}}{c^{3}}+\frac{r^{2} y^{2}}{c}
\end{array}\right) \text {. }
$$

2. Up to an affine isomorphism, there is two quadratic contravariant pseudo-Hessian structures on $\mathbb{R}^{2}$ with the divergence equivalent to the Jordan form $\left(\begin{array}{cc}a & 1 \\ 0 & a\end{array}\right)$,

$$
h_{1}=\left(\begin{array}{cc}
c y^{2}+x y & 0 \\
0 & 0
\end{array}\right) \quad \text { and } \quad h_{2}=\left(\begin{array}{cc}
\frac{1}{2} x y+c y^{2} & \frac{y^{2}}{4} \\
\frac{y^{2}}{4} & 0
\end{array}\right) .
$$

3. Up to an affine isomorphism, there is five quadratic contravariant pseudo-Hessian structures on $\mathbb{R}^{2}$ with diagonalizable divergence

$$
h_{1}=\left(\begin{array}{cc}
a x^{2} & 0 \\
0 & b y^{2}
\end{array}\right), h_{2}=\left(\begin{array}{cc}
a x^{2}+b y^{2} & 0 \\
0 & 0
\end{array}\right), h_{3}=\left(\begin{array}{cc}
a x^{2} & a x y \\
a x y & a y^{2}
\end{array}\right),
$$




$$
h_{4}=\left(\begin{array}{cc}
\frac{2 r^{2} x^{2}}{c}-2 r x y+c y^{2} & r y^{2} \\
r y^{2} & \frac{2 r^{2} y^{2}}{c}
\end{array}\right) \quad \text { and } \quad h_{5}=\left(\begin{array}{cc}
\left(\frac{2 p^{2}}{u}+\frac{q}{2}\right) x^{2}+\frac{p q x y}{u}+\frac{q^{2} y^{2}}{4 u} & p x^{2}+q x y-\frac{p q y^{2}}{2 u} \\
p x^{2}+q x y-\frac{p q y^{2}}{2 u} & \left(\frac{2 p^{2}}{u}+\frac{q}{2}\right) y^{2}+u x^{2}-2 p x y
\end{array}\right) .
$$

4. Up to an affine isomorphism, there is a unique quadratic pseudo-Hessian structure on $\mathbb{R}^{2}$ with the divergence having non real eigenvalues

$$
h=\left(\begin{array}{cc}
-2 p x y-u x^{2}+u y^{2} & p x^{2}-p y^{2}-2 u x y \\
p x^{2}-p y^{2}-2 u x y & 2 p x y+u x^{2}-u y^{2}
\end{array}\right) \text {. }
$$

Example 5.2. The study of quadratic contravariant pseudo-Hessian structures on $\mathbb{R}^{3}$ is more complicated and we give here a class of quadratic pseudo-Hessian structures on $\mathbb{R}^{3}$ of the form $\widetilde{A} \odot \widetilde{\mathrm{I}}_{3}$ where $\widetilde{A}$ is linear.

1. A is diagonal:

$$
h_{1}=\left(\begin{array}{ccc}
x^{2} & x y & x z \\
x y & y^{2} & y z \\
x z & y z & z^{2}
\end{array}\right) \text { and } h_{2}=\left(\begin{array}{ccc}
x^{2} & x y & 0 \\
x y & y^{2} & 0 \\
0 & 0 & -z^{2}
\end{array}\right)
$$

2. $A=\left(\begin{array}{lll}a & 1 & 0 \\ 0 & a & 0 \\ 0 & 0 & b\end{array}\right)$

$$
\begin{aligned}
& h_{3}=\left(\begin{array}{ccc}
2 x(y-p x) & (y-p x) y+p y x & p x z+(y-p x) z \\
(y-p x) y+p y x & 2 p y^{2} & 2 p y z \\
p x z+(y-p x) z & 2 p y z & 2 p z^{2}
\end{array}\right), \\
& h_{4}=\left(\begin{array}{ccc}
2 x(y+p x) & (y+p x) y-p y x & p x z+(y+p x) z \\
(y+p x) y+p y x & -2 p y^{2} & 0 \\
p x z+(y+p x) z & 0 & 2 p z^{2}
\end{array}\right) .
\end{aligned}
$$

\section{Right-invariant contravariant pseudo-Hessian structures on Lie groups}

Let $(\mathfrak{g}, \bullet)$ be a left symmetric algebra, i.e., for any $u,, v, w \in \mathfrak{g}$,

$$
\operatorname{ass}(u, v, w)=\operatorname{ass}(v, u, w) \quad \text { and } \quad \operatorname{ass}(u, v, w)=(u \bullet v) \bullet w-u \bullet(v \bullet w) .
$$

This implies that $[u, v]=u \bullet v-v \bullet u$ is a Lie bracket on $\mathfrak{g}$ and $\mathrm{L}: \mathfrak{g} \rightarrow \operatorname{End}(\mathfrak{g}), u \mapsto \mathrm{L}_{u}$ is a representation of the Lie algebra $(\mathfrak{g},[]$,$) . We denote by \mathrm{L}_{u}$ the left multiplication by $u$.

We consider a connected Lie group $G$ whose Lie algebra is $(\mathfrak{g},[]$,$) and we define on G$ a right invariant connection by

$$
\nabla_{u^{-}} v^{-}=-(u \bullet v)^{-},
$$

where $u^{-}$is the right vector field associated to $u \in \mathfrak{g}$. This connection is torsionless and without curvature and hence $(G, \nabla)$ is an affine manifold. Let $r \in \mathfrak{g} \otimes \mathfrak{g}$ which is symmetric and let $r^{-}$be the associated right invariant symmetric bivector field.

Proposition 6.1. $\left(G, \nabla, r^{-}\right)$is a contravariant pseudo-Hessian manifold if and only if, for any $\alpha, \beta, \gamma \in \mathfrak{g}^{*}$,

$$
[[r, r]](\alpha, \beta, \gamma):=<\gamma, r_{\#}\left([\alpha, \beta]_{r}\right)-\left[r_{\#}(\alpha), r_{\#}(\beta)\right]>=0,
$$


where

$$
[\alpha, \beta]_{r}=\mathrm{L}_{r_{*}(\alpha)}^{*} \beta-\mathrm{L}_{r_{*}(\beta)}^{*} \alpha \quad \text { and } \quad\left\langle\mathrm{L}_{u}^{*} \alpha, v>=-\langle\alpha, u \bullet v>.\right.
$$

In this case, the product on $\mathfrak{g}$ given by $\alpha . \beta=\mathrm{L}_{r_{*}(\alpha)}^{*} \beta$ is left symmetric, $[,]_{r}$ is a Lie bracket and $r_{\#}$ is a morphism of Lie algebras.

Proof. Note first that for any $\alpha \in \mathfrak{g}^{*}, r_{\#}^{-}\left(\alpha^{-}\right)=\left(r_{\#}(\alpha)\right)^{-}$and $\nabla_{u^{-}} \alpha^{-}=-\left(\mathrm{L}_{u}^{*} \alpha\right)^{-}$and hence, for any $\alpha, \beta, \gamma \in \mathfrak{g}^{*}$,

$$
\nabla_{r_{\#}^{-}\left(\alpha^{-}\right)}\left(r^{-}\right)\left(\beta^{-}, \gamma^{-}\right)=r\left(\mathrm{~L}_{r_{*}(\alpha)}^{*} \beta, \gamma\right)+r\left(\beta, \mathrm{L}_{r_{*}(\alpha)}^{*} \gamma\right) .
$$

So, $\left(G, \nabla, r^{-}\right)$is a contravariant pseudo-Hessian manifold if and only if, for any $\alpha, \beta, \gamma \in \mathfrak{g}^{*}$,

$$
\begin{aligned}
0 & =r\left(\mathrm{~L}_{r_{\#}(\alpha)}^{*} \beta, \gamma\right)+r\left(\beta, \mathrm{L}_{r_{*}(\alpha)}^{*} \gamma\right)-r\left(\mathrm{~L}_{r_{\#}(\beta)}^{*} \alpha, \gamma\right)-r\left(\alpha, \mathrm{L}_{r_{\#}(\beta)}^{*} \gamma\right) \\
& =\left\langle\gamma, r_{\#}\left([\alpha, \beta]_{r}\right)-r_{\#}(\alpha) \bullet r_{\#}(\beta)+r_{\#}(\beta) \bullet r_{\#}(\alpha)>\right. \\
& =\left\langle\gamma, r_{\#}\left([\alpha, \beta]_{r}\right)-\left[r_{\#}(\alpha), r_{\#}(\beta)\right]\right\rangle
\end{aligned}
$$

and the first part of the proposition follows. Suppose now that $r_{\#}\left([\alpha, \beta]_{r}\right)=\left[r_{\#}(\alpha), r_{\#}(\beta)\right]$ for any $\alpha, \beta \in \mathfrak{g}^{*}$. Then, for any $\alpha, \beta, \gamma \in \mathfrak{g}^{*}$,

$$
\operatorname{ass}(\alpha, \beta, \gamma)-\operatorname{ass}(\beta, \alpha, \gamma)=\mathrm{L}_{r_{*}([\alpha, \beta] r)}^{*} \gamma-\mathrm{L}_{r_{*}(\alpha)}^{*} \mathrm{~L}_{r_{\sharp}(\beta)}^{*} \gamma+\mathrm{L}_{r_{*}(\beta)}^{*} \mathrm{~L}_{r_{*}(\alpha)}^{*} \gamma=0 .
$$

This completes the proof.

Definition 6.2. 1. Let $(\mathfrak{g}, \bullet)$ be a left symmetric algebra. A symmetric bivector $r \in \mathfrak{g} \otimes \mathfrak{g}$ satisfying $[[r, r]]=0$ is called a $S$-matrix.

2. A left symmetric algebra $(\mathfrak{g}, \bullet, r)$ endowed with a $S$-matrix is called a contravariant pseudoHessian algebra.

Let $(\mathfrak{g}, \bullet, r)$ be a contravariant pseudo-Hessian algebra, $[u, v]=u \bullet v-v \bullet u$ and $G$ a connected Lie group with $(\mathfrak{g},[]$,$) as a Lie algebra. We have shown that G$ carries a right invariant contravariant pseudo-Hessian structure $\left(\nabla, r^{-}\right)$. On the other hand, in Section 3, we have associated to $\left(\nabla, r^{-}\right)$a flat connection $\bar{\nabla}$, a complex structure $J$ and a Poisson tensor $\Pi$ on $T G$. Now we will show that $T G$ carries a structure of Lie group and the triple $(\bar{\nabla}, J, \Pi)$ is right invariant. This structure of Lie group on $T G$ is different from the usual one defined by the adjoint action of $G$ on $\mathfrak{g}$.

Let us start with a general algebraic construction which is interesting on its own. Let $(\mathfrak{g}, \bullet)$ be a left symmetric algebra, put $\Phi(\mathfrak{g})=\mathfrak{g} \times \mathfrak{g}$ and define a product $\star$ and a bracket on $\Phi(\mathfrak{g})$ by

$$
(a, b) \star(c, d)=(a \bullet c, a \bullet d) \quad \text { and } \quad[(a, b),(c, d)]=([a, c], a \bullet d-c \bullet b),
$$

for any $(a, b),(c, d) \in \Phi(\mathrm{g})$. It is easy to check that $\star$ is left symmetric, [ , ] is the commutator of $\star$ and hence is a Lie bracket. We define also $J_{0}: \Phi(\mathfrak{g}) \longrightarrow \Phi(\mathfrak{g})$ by $J_{0}(a, b)=(b,-a)$. It is also a straightforward computation to check that

$N_{J_{0}}((a, b),(c, d))=\left[J_{0}(a, b), J_{0}(c, d)\right]-J_{0}\left[(a, b), J_{0}(c, d)\right]-J_{0}\left[J_{0}(a, b),(c, d)\right]-[(a, b),(c, d)]=0$.

For $r \in \otimes^{2} \mathfrak{g}$ symmetric, we define $R \in \otimes^{2} \Phi(\mathfrak{g})$ by

$$
R\left(\left(\alpha_{1}, \beta_{1}\right),\left(\alpha_{2}, \beta_{2}\right)\right)=r\left(\alpha_{1}, \beta_{2}\right)-r\left(\alpha_{2}, \beta_{1}\right),
$$

for any $\alpha_{1}, \alpha_{2}, \beta_{1}, \beta_{2} \in \mathrm{g}^{*}$. We have obviously that $R_{\#}\left(\alpha_{1}, \beta_{1}\right)=\left(-r_{\#}\left(\beta_{1}\right), r_{\#}\left(\alpha_{1}\right)\right)$. 
Proposition 6.3. $[[r, r]]=0$ if and only if $[R, R]=0$, where $[R, R]$ is the Schouten bracket associated to the Lie algebra structure of $\Phi(\mathfrak{g})$ and given by

$$
[R, R](\alpha, \beta, \gamma)=\oint_{\alpha, \beta, \gamma} \prec \gamma,\left[R_{\#}(\alpha), R_{\#}(\beta)\right]>, \quad \alpha, \beta, \gamma \in \Phi^{*}(\mathfrak{g}) .
$$

Proof. For any $\alpha=\left(\alpha_{1}, \alpha_{2}\right), \beta=\left(\beta_{1}, \beta_{2}\right), \gamma=\left(\gamma_{1}, \gamma_{2}\right) \in \Phi(\mathfrak{g})^{*}$,

$$
\begin{aligned}
\prec \gamma,\left[R_{\#}(\alpha), R_{\#}(\beta)\right]= & <\left(\gamma_{1}, \gamma_{2}\right),\left[\left(-r_{\#}\left(\alpha_{2}\right), r_{\#}\left(\alpha_{1}\right)\right),\left(-r_{\#}\left(\beta_{2}\right), r_{\#}\left(\beta_{1}\right)\right)\right]> \\
= & <\gamma_{1},\left[r_{\#}\left(\alpha_{2}\right), r_{\#}\left(\beta_{2}\right)\right]>-\prec \gamma_{2}, r_{\#}\left(\alpha_{2}\right) \bullet r_{\#}\left(\beta_{1}\right)>+\prec \gamma_{2}, r_{\#}\left(\beta_{2}\right) \bullet r_{\#}\left(\alpha_{1}\right)> \\
= & <\gamma_{1},\left[r_{\#}\left(\alpha_{2}\right), r_{\#}\left(\beta_{2}\right)\right]>+<\beta_{1}, r_{\#}\left(\mathrm{~L}_{r_{\#}\left(\alpha_{2}\right)}^{*} \gamma_{2}\right)>-\prec \alpha_{1}, r_{\#}\left(\mathrm{~L}_{r_{\#}\left(\beta_{2}\right)}^{*} \gamma_{2}\right)>, \\
\prec \beta,\left[R_{\#}(\gamma), R_{\#}(\alpha)\right]>= & <\beta_{1},\left[r_{\#}\left(\gamma_{2}\right), r_{\#}\left(\alpha_{2}\right)\right]>+<\alpha_{1}, r_{\#}\left(\mathrm{~L}_{r_{\#}\left(\gamma_{2}\right)}^{*} \beta_{2}\right)>-\prec \gamma_{1}, r_{\#}\left(\mathrm{~L}_{r_{\#}\left(\alpha_{2}\right)}^{*} \beta_{2}\right)> \\
<\alpha,\left[R_{\#}(\beta), R_{\#}(\gamma)\right]>= & <\alpha_{1},\left[r_{\#}\left(\beta_{2}\right), r_{\#}\left(\gamma_{2}\right)\right]>+\prec \gamma_{1}, r_{\#}\left(\mathrm{~L}_{r_{\#}\left(\beta_{2}\right)}^{*} \alpha_{2}\right)>-\prec \beta_{1}, r_{\#}\left(\mathrm{~L}_{r_{\#}\left(\gamma_{2}\right)}^{*} \alpha_{2}\right)>.
\end{aligned}
$$

So

$$
[R, R](\alpha, \beta, \gamma)=-[[r, r]]\left(\beta_{2}, \gamma_{2}, \alpha_{1}\right)-[[r, r]]\left(\gamma_{2}, \alpha_{2}, \beta_{1}\right)-[[r, r]]\left(\alpha_{2}, \beta_{2}, \gamma_{1}\right)
$$

and the result follows.

Let $G$ be a Lie group whose Lie algebra is $(\mathfrak{g},[]$,$) and let \rho: G \longrightarrow$ GL(g) be the homomorphism of groups such that $d_{e} \rho=\mathrm{L}$ where $\mathrm{L}: \mathfrak{g} \longrightarrow \operatorname{End}(\mathfrak{g})$ is the representation associated to $\bullet$. Then the product

$$
(g, u) .(h, v)=(g h, u+\rho(g)(v)), \quad g, h \in G, u, v \in \mathfrak{g}
$$

induces a Lie group structure on $G \times \mathfrak{g}$ whose Lie algebra is $(\Phi(\mathfrak{g}),[$, ]). The complex endomorphism $J_{0}$ and the left symmetric product $\star$ induce a right invariant complex tensor $J_{0}^{-}$and a a right invariant connection $\widetilde{\nabla}$ given by

$$
J_{0}^{-}(a, b)^{-}=(b,-a)^{-} \quad \text { and } \quad \widetilde{\nabla}_{(a, b)^{-}}(c, d)^{-}=-((a, b) \star(c, d))^{-} .
$$

Let $r \in \otimes^{2} \mathrm{~g}$ symmetric such that $[[r, r]]=0, r^{-}$the associated right invariant symmetric bivector field and $\nabla$ the affine connection given by (6.1). Then $\left(G, \nabla, r^{-}\right)$is a contravariant pseudo-Hessian manifold and let $\bar{\nabla}, J$ and $\Pi$ be the associated structure on $T G$ defined in Section 3

Theorem 6.4. If we identify $T G$ with $G \times \mathfrak{g}$ by $u_{g} \longrightarrow\left(g, T_{g} R_{g^{-1}} u_{g}\right)$ we denote also by $\Pi, \bar{\nabla}$ and $J$ the images of $\Pi, \bar{\nabla}$ and $J$ under this identification then $\Pi=R^{-}, \bar{\nabla}=\widetilde{\nabla}$ and $J=J_{0}^{-}$.

To prove this theorem, we need some preparation.

Proposition 6.5. Let $(G, \nabla)$ be a Lie group endowed with a right invariant connection and $\gamma$ : $[0,1] \longrightarrow G$ a curve. Let $V:[0,1] \longrightarrow T G$ be a vector field along $\gamma$. We define $\mu:[0,1] \longrightarrow \mathfrak{g}$ and $W:[0,1] \longrightarrow \mathfrak{g}$ by

$$
\mu(t)=T_{\gamma(t)} R_{\gamma(t)^{-1}}\left(\gamma^{\prime}(t)\right) \quad \text { and } \quad W(t)=T_{\gamma(t)} R_{\gamma(t)^{-1}}(V(t)) .
$$

Then $V$ is parallel along $\gamma$ with respect $\nabla$ if and only if

$$
W^{\prime}(t)-\mu(t) \bullet W(t)=0,
$$

where $u \bullet v=-\left(\nabla_{u^{-}} v^{-}\right)(e)$. 
Proof. We consider $\left(u_{1}, \ldots, u_{n}\right)$ a basis of $\mathfrak{g}$ and $\left(X_{1}, \ldots, X_{n}\right)$ the corresponding right invariant vector fields. Then

$$
\left\{\begin{array}{l}
\mu(t)=\sum_{i=1}^{n} \mu_{i}(t) u_{i}, W(t)=\sum_{i=1}^{n} W_{i}(t) u_{i}, \\
\gamma^{\prime}(t)=\sum_{i=1}^{n} \mu_{i}(t) X_{i}, V(t)=\sum_{i=1}^{n} W_{i}(t) X_{i} .
\end{array}\right.
$$

Then

$$
\begin{aligned}
\nabla_{t} V(t) & =\sum_{i=1}^{n} W_{i}^{\prime}(t) X_{i}+\sum_{i=1}^{n} W_{i}(t) \nabla_{\gamma^{\prime}(t)} X_{i} \\
& =\sum_{i=1}^{n} W_{i}^{\prime}(t) X_{i}+\sum_{i, j=1}^{n} W_{i}(t) \mu_{j}(t) \nabla_{X_{j}} X_{i} \\
& =\sum_{i=1}^{n} W_{i}^{\prime}(t) X_{i}-\sum_{i, j=1}^{n} W_{i}(t) \mu_{j}(t)\left(u_{j} \bullet u_{i}\right)^{-} \\
& =\left(W^{\prime}(t)-\mu(t) \bullet W(t)\right)^{-}
\end{aligned}
$$

and the result follows having in mind that $u^{-}$is the right invariant vector field associated to $u \in \mathfrak{g}$.

Let $(G, \nabla)$ be a Lie group endowed with a right invariant connection. Then $\nabla$ induces a splitting of $T T G=\operatorname{ker} d p \oplus \mathcal{H}$. For any tangent vector $X \in T_{g} G$, we denote by $X^{v}, X^{h} \in T_{(g, u)} T G$ the vertical and the horizontal lift of $X$.

Proposition 6.6. If we identify $T G$ to $G \times \mathfrak{g}$ by $X_{g} \mapsto\left(g, T_{g} R_{g^{-1}}\left(X_{g}\right)\right)$ then for any $X \in T_{g} G$,

$$
X^{v}(g, u)=\left(0, T_{g} R_{g^{-1}}(X)\right) \quad \text { and } \quad X^{h}(g, u)=\left(X, T_{g} R_{g^{-1}}(X) \bullet u\right) .
$$

Proof. The first relation is obvious. Recall that the horizontal lift of $X$ at $u_{g} \in T G$ is given by:

$$
X^{h}\left(u_{g}\right)=\frac{d}{d t}{ }_{t=0} V(t)
$$

where $V:[0,1] \longrightarrow T G$ is the parallel vector field along $\gamma:[0,1] \longrightarrow G$ a curve such that $\gamma(0)=g$ and $\gamma^{\prime}(0)=X$. If we denote by $\Theta_{R}: T G \longrightarrow G \times \mathfrak{g}$ the identification $u_{g} \mapsto\left(g, T_{g} R_{g^{-1}}\left(u_{g}\right)\right)$ then, by virtue of Proposition 6.5 .

$$
T_{u_{g}} \Theta_{R}\left(X^{h}\right)=\left.\frac{d}{d t}\right|_{t=0}(\gamma(t), W(t))=\left(X, T_{g} R_{g^{-1}}(X) \bullet u\right) .
$$

We consider now a left symmetric algebra $(\mathfrak{g}, \bullet), G$ a connected Lie group associated to ( $\mathfrak{g},[],), \nabla$ the right invariant affine connection associated to $\bullet$. We have seen that $G \times \mathfrak{g}$ has a structure of Lie group. We identify $T G$ to $G \times \mathfrak{g}$ and, for any vector field $X$ on $G$, we denote by $X^{v}$ and $X^{h}$ the vector fields on $G \times \mathfrak{g}$ obtained by the identification from the horizontal and the vertical lift of $X$. For $a, b \in \mathfrak{g}, \alpha, \beta \in \mathfrak{g}^{*}, a^{-}$(resp. $\alpha^{-}$) is the right invariant vector field (resp. 1 -form) on $G$ associated to $a$ (resp. $\alpha$ ), $(a, b)^{-}$(resp. $\left.(\alpha, \beta)^{-}\right)$the right invariant vector field (resp. 1 -form) on $G \times \mathfrak{g}$ associated to $(a, b)$ (resp. $(\alpha, \beta))$. 
Proposition 6.7. For any $(a, b) \in \mathfrak{g} \times \mathfrak{g}$ and $(\alpha, \beta) \in \mathfrak{g}^{*} \times \mathfrak{g}^{*}$,

$$
(a, b)^{-}=\left(a^{-}\right)^{h}+\left(b^{-}\right)^{v} \text { and }(\alpha, \beta)^{-}=\left(\alpha^{-}\right)^{h}+\left(\beta^{-}\right)^{v} .
$$

Proof. We have

$$
\begin{aligned}
(a, b)^{-}(g, u) & =T_{(e, 0)} R_{(g, u)}(a, b) \\
& =\frac{d}{d t}_{\mid t=0}(\exp (t a), t b)(g, u) \\
& =\frac{d}{d t}_{t=0}(\exp (t a) g, t b+\rho(\exp (t a))(u)) \\
& =\left(a^{-}(g), b+a \bullet u\right) \\
& =\left(a^{-}(g), T_{g} R_{g^{-1}}\left(a^{-}(g)\right) \bullet u\right)+\left(0, T_{g} R_{g^{-1}}\left(b^{-}(g)\right)\right. \\
& =\left(a^{-}\right)^{h}(g, u)+\left(b^{-}\right)^{v}(g, u) . \quad(\text { Proposition 6.5) }
\end{aligned}
$$

The second relation can be deduced easily from the first one.

\section{Proof of Theorem 6.4 .}

Proof. Let $\Pi$ be the Poisson tensor on $G \times \mathfrak{g}$ associated to $r^{-}$. Then, by using the precedent proposition,

$$
\begin{aligned}
\Pi\left(\left(\alpha_{1}, \beta_{1}\right)^{-},\left(\alpha_{2}, \beta_{2}\right)^{-}\right) & =\Pi\left(\left(\alpha_{1}^{-}\right)^{h}+\left(\beta_{1}^{-}\right)^{v},\left(\alpha_{2}^{-}\right)^{h}+\left(\beta_{2}^{-}\right)^{v}\right) \\
& =r^{-}\left(\alpha_{1}^{-}, \beta_{2}^{-}\right)-r^{-}\left(\alpha_{2}^{-}, \beta_{1}^{-}\right) \\
& =r\left(\alpha_{1}, \beta_{2}\right)-r\left(\alpha_{2}, \beta_{1}\right) \\
& =R^{-}\left(\left(\alpha_{1}, \beta_{1}\right)^{-},\left(\alpha_{2}, \beta_{2}\right)^{-}\right) .
\end{aligned}
$$

In the same way,

$$
\begin{aligned}
J_{0}^{-}(a, b)^{-} & =(b,-a)^{-}=\left(b^{-}\right)^{h}-\left(a^{-}\right)^{v} \\
J(a, b)^{-} & =\left(b^{-}\right)^{h}-\left(a^{-}\right)^{v} \\
\bar{\nabla}_{(a, b)^{-}}(c, d)^{-} & =\left(\nabla_{a^{-}} c^{-}\right)^{h}+\left(\nabla_{a^{-}} d^{-}\right)^{v}=-\left((a \bullet c)^{-}\right)^{h}-\left((a \bullet d)^{-}\right)^{v}=-((a, b) .(c, d))^{-}=\widetilde{\nabla}_{(a, b)^{-}}(c, d)^{-} .
\end{aligned}
$$

Let $(\mathfrak{g}, \bullet)$ be a left symmetric algebra, $(M, \nabla)$ and affine manifold and $\rho: \mathfrak{g} \longrightarrow \Gamma(T M)$ a linear map such that $\rho(u \bullet v)=\nabla_{\rho(u)} \rho(v)$. Then $\rho$ defines an action on $M$ of the Lie algebra $(\mathfrak{g},[]$,$) . We consider \rho^{l}: \Phi(\mathfrak{g}) \longrightarrow \Gamma(T T M),(u, v) \longrightarrow \rho(u)^{h}+\rho^{v}(v)$. It is easy to check that

$$
\rho^{l}([a, b])=\left[\rho^{l}(a), \rho^{l}(b)\right] .
$$

Let $r \in \otimes^{2} \mathfrak{g}$ satisfying $[[r, r]]=0$ and $R \in \otimes^{2} \Phi(\mathfrak{g})$ given by $\underline{6.3}$ ).

Theorem 6.8. The bivector field on TM associated to $\rho(r)$ is $\rho^{l}(R)$ which is a Poisson tensor and $(M, \nabla, \rho(r))$ is a contravariant pseudo-Hessian manifold. 
Proof. Let $\left(e_{1}, \ldots, e_{n}\right)$ a basis of $\mathfrak{g}$ and $E_{i}=\left(e_{i}, 0\right)$ and $F_{i}=\left(0, e_{i}\right)$. Then $\left(E_{1}, \ldots, E_{n}, F_{1}, \ldots, F_{n}\right)$ is a basis of $\Phi(\mathrm{g})$. Then

$$
r=\sum_{i, j} r_{i, j} e_{i} \otimes e_{j} \quad \text { and } \quad R=\sum_{i, j} r_{i, j}\left(E_{i} \otimes F_{j}-F_{i} \otimes E_{j}\right) .
$$

So

$$
\rho(r)=\sum_{i, j=1}^{n} r_{i, j} \rho\left(e_{i}\right) \otimes \rho\left(e_{j}\right) \quad \text { and } \quad \rho^{l}(R)=\sum_{i, j=1}^{n} r_{i, j}\left(\rho\left(e_{i}\right)^{h} \otimes \rho\left(e_{j}\right)^{v}-\rho\left(e_{i}\right)^{v} \otimes \rho\left(e_{j}\right)^{h}\right) .
$$

Then for any $\alpha, \beta \in \Omega^{1}(M)$

$$
\rho^{l}(R)\left(\alpha^{v}, \beta^{v}\right)=\rho^{l}(R)\left(\alpha^{h}, \beta^{h}\right)=0 \quad \text { and } \quad \rho^{l}(R)\left(\alpha^{h}, \beta^{v}\right)=\rho(r)(\alpha, \beta) \circ p .
$$

According to Proposition 6.3, $R$ is a solution of the classical Yang-Baxer equation and hence $\rho^{l}(R)$ is a Poisson tensor. By using Theorem 3.3 , we get that $(M, \nabla, \rho(r))$ is a contravariant pseudo-Hessian manifold.

Example 6.9. 1. Let $\mathrm{g}=\mathrm{gl}(n, \mathbb{R})$ be the Lie algebra of $n$-square matrices. It is has a structure of left symmetric algebra given by $A \bullet B=B A$. Let $\rho: \mathfrak{g} \longrightarrow \Gamma\left(T \mathbb{R}^{n}\right)$ given by $\rho(A)=A$. Then $\rho(A \bullet B)=\nabla_{A} B$, where $\nabla$ is the canonical connection of $\mathbb{R}$. According to Theorem 6.8 any $S$-matrix on g gives rise to a quadratic contravariant pseudo-Hessian structure on $\mathbb{R}^{n}$.

2. More generally, let $(M, \nabla)$ be an affine manifold and $\mathrm{g}$ the finite dimensional Lie algebra of affine vector fields. Recall that $X \in \mathfrak{g}$ if for any $Y, Z \in \Gamma(T M)$,

$$
\left[X, \nabla_{Y} Z\right]=\nabla_{[X, Y]} Z+\nabla_{Y}[X, Z] .
$$

Since the curvature and the torsion of $\nabla$ vanish this is equivalent to

$$
\nabla_{\nabla_{Y} Z} X=\nabla_{Y} \nabla_{Z} X
$$

From this relation, one can see easily that, for any $X, Y \in \mathfrak{g}, X \bullet Y:=\nabla_{X} Y \in \mathfrak{g}$ and $(\mathfrak{g}, \bullet)$ is an associative finite dimensional Lie algebra which acts on $M$ by $\rho(X)=X$. Moreover, $\rho(X \bullet Y)=\nabla_{X} Y$. According to Theorem 6.8 any $S$-matrix on gives rise to a contravariant pseudo-Hessian structure on $M$.

\section{Classification of two-dimensional contravariant pseudo-Hessian algebras}

Using the classification of two-dimensional non-abelian left symmetric algebras given in [5] and the classification of abelian left symmetric algebras given in [14], we give a classification (over the field $\mathbb{R}$ ) of 2-dimensional contravariant pseudo-Hessian algebras. We proceed as follows:

1. For any left symmetric 2-dimensional algebra $\mathfrak{g}$, we determine its automorphism group Aut $(\mathfrak{g})$ and the space of $S$-matrices on $\mathfrak{g}$, we denote by $\mathcal{A}(\mathfrak{g})$.

2. We give the quotient $\mathcal{A}(\mathrm{g}) / \sim$ where $\sim$ is the equivalence relation:

$$
r^{1} \sim r^{2} \Longleftrightarrow \exists A \in \operatorname{Aut}(\mathrm{g}) \text { or } \exists \lambda \in \mathbb{R} \text { such that } r_{\sharp}^{2}=A \circ r_{\sharp}^{1} \circ A^{t} \text { or } r^{2}=\lambda r^{1} .
$$




\begin{tabular}{|c|c|c|}
\hline$(\mathfrak{g},)$. & Aut $(\mathfrak{g})$ & $\mathcal{A}(\mathfrak{g}) / \sim$ \\
\hline $\begin{array}{l}b_{1, \alpha \neq-1,1} \\
e_{2} . e_{1}=e_{1}, e_{2} \cdot e_{2}=\alpha e_{2}\end{array}$ & $\left(\begin{array}{ll}a & 0 \\
0 & 1\end{array}\right), a \neq 0$ & $r_{\sharp}^{1}=\left(\begin{array}{ll}1 & 0 \\
0 & 0\end{array}\right) ; r_{\sharp}^{2}=\left(\begin{array}{ll}0 & 0 \\
0 & 1\end{array}\right) ; r_{\sharp}^{3}=0$ \\
\hline $\begin{array}{l}b_{1, \alpha=-1} \\
e_{2} \cdot e_{1}=e_{1}, e_{2} \cdot e_{2}=-e_{2}\end{array}$ & $\left(\begin{array}{ll}a & 0 \\
0 & 1\end{array}\right), a \neq 0$ & $\begin{array}{l}r_{\sharp}^{1}=\left(\begin{array}{ll}b & 1 \\
1 & 0\end{array}\right) ; r_{\sharp}^{2}=\left(\begin{array}{ll}1 & 0 \\
0 & 0\end{array}\right) ; \\
r_{\sharp}^{3}=\left(\begin{array}{ll}0 & 0 \\
0 & 1\end{array}\right) ; r_{\sharp}^{4}=0\end{array}$ \\
\hline $\begin{array}{l}b_{1, \alpha=1} \\
e_{2} \cdot e_{1}=e_{1}, e_{2} \cdot e_{2}=e_{2}\end{array}$ & $\left(\begin{array}{ll}a & 0 \\
0 & b\end{array}\right), a b \neq 0$ & $r_{\sharp}^{1}=\left(\begin{array}{cc}1 & c \\
c & c^{2}\end{array}\right) ; r_{\sharp}^{2}=\left(\begin{array}{ll}0 & 0 \\
0 & 1\end{array}\right) ; r_{\#}^{3}=0$ \\
\hline $\begin{array}{l}b_{2, \beta \neq 0,1,2} \\
e_{1} \cdot e_{2}=\beta e_{1}, e_{2} \cdot e_{1}=(\beta- \\
1) e_{1}, e_{2} \cdot e_{2}=\beta e_{2}\end{array}$ & $\left(\begin{array}{ll}a & b \\
0 & 1\end{array}\right), a \neq 0$ & $r_{\sharp}^{1}=\left(\begin{array}{ll}1 & 0 \\
0 & 0\end{array}\right) ; r_{\sharp}^{2}=\left(\begin{array}{ll}0 & 0 \\
0 & 1\end{array}\right) ; r_{\sharp}^{3}=0$ \\
\hline $\begin{array}{l}b_{2, \beta=1} \\
e_{1} \cdot e_{2}=e_{1}, e_{2} \cdot e_{2}=e_{2}\end{array}$ & $\left(\begin{array}{ll}a & b \\
0 & 1\end{array}\right), a \neq 0$ & $r_{\sharp}^{1}=\left(\begin{array}{cc}1 & c \\
c & c^{2}\end{array}\right) ; r_{\sharp}^{2}=\left(\begin{array}{ll}0 & 0 \\
0 & 1\end{array}\right) ; r_{\sharp}^{3}=0$ \\
\hline $\begin{array}{l}b_{2, \beta=2} \\
e_{1} \cdot e_{2}=2 e_{1}, \\
e_{2} \cdot e_{1}=e_{1}, e_{2} \cdot e_{2}=2 e_{2}\end{array}$ & $\left(\begin{array}{ll}a & b \\
0 & 1\end{array}\right), a \neq 0$ & $r_{\sharp}^{1}=\left(\begin{array}{ll}1 & 0 \\
0 & c\end{array}\right) ; r_{\sharp}^{2}=\left(\begin{array}{ll}0 & 0 \\
0 & 1\end{array}\right) ; r_{\sharp}^{3}=0$ \\
\hline $\begin{array}{l}b_{3} \\
e_{2} . e_{1}=e_{1}, e_{2} . e_{2}=e_{1}+e_{2}\end{array}$ & $\left(\begin{array}{ll}1 & b \\
0 & 1\end{array}\right)$ & $r_{\sharp}^{1}=\left(\begin{array}{cc}1 / 2 & 1 \\
1 & 1\end{array}\right) ; r_{\sharp}^{2}=\left(\begin{array}{ll}1 & 0 \\
0 & 0\end{array}\right) ; r_{\sharp}^{3}=0$ \\
\hline $\begin{array}{l}b_{4} \\
e_{1} \cdot e_{1}=2 e_{1}, e_{1} \cdot e_{2}=e_{2} \\
e_{2} \cdot e_{2}=e_{1}\end{array}$ & $\left(\begin{array}{cc}1 & 0 \\
0 & -1\end{array}\right) ;\left(\begin{array}{ll}1 & 0 \\
0 & 1\end{array}\right)$ & $r_{\#}^{1}=\left(\begin{array}{ll}1 & 0 \\
0 & 2\end{array}\right) ; r_{\sharp}^{2}=\left(\begin{array}{ll}1 & 0 \\
0 & 0\end{array}\right) ; r_{\sharp}^{3}=0$ \\
\hline $\begin{array}{l}b_{5} \\
e_{1} \cdot e_{2}=e_{1}, e_{2} \cdot e_{2}=e_{1}+e_{2}\end{array}$ & $\left(\begin{array}{ll}1 & b \\
0 & 1\end{array}\right)$ & $r_{\sharp}^{1}=\left(\begin{array}{ll}1 & 0 \\
0 & 0\end{array}\right) ; r_{\sharp}^{2}=0$ \\
\hline $\begin{array}{l}A s_{2}^{1} \\
e_{1} \cdot e_{1}=e_{2}\end{array}$ & $\left(\begin{array}{cc}a & 0 \\
b & a^{2}\end{array}\right), a \neq 0$ & $r_{\sharp}^{1}=\left(\begin{array}{ll}0 & 1 \\
1 & 0\end{array}\right) ; r_{\sharp}^{2}=\left(\begin{array}{ll}0 & 0 \\
0 & 1\end{array}\right) ; r_{\sharp}^{3}=0$ \\
\hline
\end{tabular}

\begin{tabular}{|l|l|l|}
\hline$A s_{2}^{4}$ & $\left(\begin{array}{ll}1 & 0 \\
0 & a\end{array}\right), a \neq 0$ & $r_{\sharp}^{1}=\left(\begin{array}{ll}0 & 1 \\
1 & c\end{array}\right) ; r_{\sharp}^{2}=\left(\begin{array}{ll}0 & 0 \\
0 & 1\end{array}\right) ;$ \\
$e_{1} \cdot e_{1}=e_{1}, e_{1} \cdot e_{2}=e_{2}$, & & $r_{\sharp}^{3}=\left(\begin{array}{ll}1 & 0 \\
0 & 0\end{array}\right) ; r_{\sharp}^{4}=0$ \\
\hline
\end{tabular}

We end this paper by giving another proof to Lemma 4.4 .

Proof. For any $\gamma:[0,1] \longrightarrow G \times G, t \mapsto\left(\gamma_{1}(t), \gamma_{2}(t)\right)$ with $\gamma(0)=(a, b)$ and $\gamma(1)=(c, d)$,

$$
\tau_{m(\gamma)}\left(T_{(a, b)} m(u, v)\right)=T_{(c, d)} m\left(\tau_{\gamma}(u, v)\right),
$$

where $\tau_{\gamma}: T_{(a, b)}(G \times G) \longrightarrow T_{(c, d)}(G \times G)$ and $\tau_{m \gamma}: T_{a b} G \longrightarrow T_{c d} G$ are the parallel transports. But

$$
T_{(a, b)} m(u, v)=T_{a} R_{b}(u)+T_{b} L_{a}(v) \quad \text { and } \quad \tau_{\gamma}(u, v)=\left(\tau_{\gamma_{1}}(u), \tau_{\gamma_{2}}(v)\right) .
$$


So we get

$$
\tau_{\gamma_{1} \gamma_{2}}\left(T_{a} R_{b}(u)\right)+\tau_{\gamma_{1} \gamma_{2}}\left(T_{b} L_{a}(v)\right)=T_{c} R_{d}\left(\tau_{\gamma_{1}}(u)\right)+T_{d} L_{c}\left(\tau_{\gamma_{2}}(v)\right) .
$$

If we take $v=0$ and $\gamma_{2}(t)=b=d$. We get

$$
\tau_{\gamma_{1} b}\left(T_{a} R_{b}(u)\right)=T_{c} R_{b}\left(\tau_{\gamma_{1}}(u)\right)
$$

and hence $\nabla$ is right invariant. In the same way we get that $\nabla$ is left invariant. And finally

$$
\tau_{\gamma_{1} \gamma_{2}}\left(T_{a} R_{b}(u)\right)=T_{c} R_{d}\left(\tau_{\gamma_{1}}(u)\right) \quad \text { and } \quad \tau_{\gamma_{1} \gamma_{2}}\left(T_{b} L_{a}(v)\right)=T_{d} L_{c}\left(\tau_{\gamma_{2}}(v)\right)
$$

If we take $\gamma_{2}=\gamma_{1}^{-1}$ we get that

$$
\tau_{\gamma_{1}}(u)=T_{a} R_{a^{-1} c}(u)=T_{a} L_{c a^{-1}}(u) .
$$

This implies that the adjoint representation is trivial and hence $G$ must be abelian.

\section{References}

[1] C. Bai. Bijective 1-cocycles and classification of 3-dimensional left-symmetric algebras, Communications in Algebra Volume 37, 1016-1057 (2009).

[2] Bai, C., Left-Symmetric Bialgebras and an Analogue of the Classical Yang-Baxter Equation, Communication in Contemporary Mathematics, 2008, Vol. 10; Numb. 2, 221-260.

[3] S. Benayadi and M. Boucetta. On para-Kähler Lie algebroids and contravariant pseudo-Hessian structures. Mathematische Nachrichten. (2019);1-26.

[4] S. Benayadi and M. Boucetta. On Para-Kähler and Hyper-Kähler Lie algebras. Journal of Algebra 436 (2015) 61-101.

[5] D. Burde Simple left-symmetric algebras with solvable Lie algebra, manuscripta math. 95, 397 - 411 (1998).

[6] C. Calvin and L. M. C. Schochet, Global analysis on foliated space. MISRP (2006).

[7] Dombrowski P., On the geometry of the tangent bundle. J. Reme Angew. Math. 210 (1962), 73-78.

[8] Dufour, Jean-Paul, and Nguyen Tien Zung. Poisson structures and their normal forms. Vol. 242. Springer Science and Business Media, 2006.

[9] J. P. Dufour and A. Haraki, Rotationnels et structures de Poisson quadratiques. C. R. Acad. Sci. Paris Série. I Math., 312(1):137-140, 1991.

[10] R. L. Fernandes, Lie algebroids, holonomy and characteristic classes, Adv. in Math. 170 (2002), 119-179.

[11] Christian Kassel, Homology and cohomology of associative algebras. A concise introduction to cyclic homology. Thematic school. August 2004 at ICTP, Trieste (Italy), 2006. ¡cel-00119891;

[12] K. Mackenzie, Lie Groupoids and Lie Algebroids in Differential Geometry, London Math. Soc. Lecture Notes Ser. 124, Cambridge Univ. Press, Cambridge, 1987.

[13] H. Shima. The geometry of Hessian structures. World Scientific Publishing (2007).

[14] I.S. Rakhimov, I.M. Rikhsiboev, W. Basri. Complete lists of low dimensional complex associative algebras, arXiv:0910.0932 2

[15] A. Weinstein, The local structure of Poisson manifolds, J. Differential Geom. Volume 18, Number 3 (1983), 523-557. 